\section{Global Proceedings Repository \\ American Research Foundation}

ISSN 2476-017X

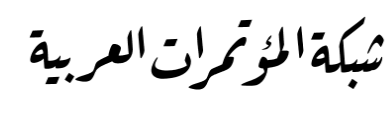

http://arab.kmshare.net/

Available online at http://proceedings.sriweb.org

The 10th International Scientific Conference

Under the Title

"Geophysical, Social, Human and Natural Challenges in a Changing Environment"

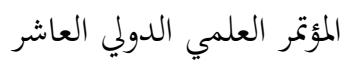

$$
\begin{aligned}
& \text { تحت عنوان "التحديات الجيوفيزيائية والاجتماعية والانسانية والطبيعية في بيئة متغيرة" } \\
& 25 \text { - } 26 \text { يوليو - تموز } 2019 \text { - اسطنبول - تركيا } \\
& \text { http://kmshare.net/isac2019/ }
\end{aligned}
$$

\title{
أهمية علاقة إدارة المعرفة بإدارة المشاريع: تكامل العمليات ونجاح المثروع
}

The importance of $(\mathrm{KM})$ relation to $(\mathrm{PM})$ :

Processes integration and project success.

\author{
عبدالسلام مُحمَّمَ الغامدي \\ دكتوراه - قسم علم المعلومات - إدارة المعرفة \\ أهمية علاقة إدارة المعرفة بإدارة المشاريع: تكامل العمليات ونجاح المشروع
}

المستخلص:

Intellectual وما تتضمنه من رأس مال بشري وفكري إدارة المعرفة Knowledge Management (KM) Capital 


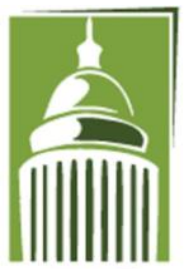

ARF
Global Proceedings Repository

American Research Foundation

ISSN 2476-017X

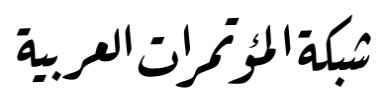

http://arab.kmshare.net/

Available online at http://proceedings.sriweb.org

نقلة نوعية في إدارة المعرفة وتبني استراتيجياتا. وبالتالي يسعى البحث الحالي إلى توضيح أهمية علاقة إدارة المعرفة بإدارة المشاريع من خلال التعرف على أثرها في تحقيق تكامل العمليات ونجاح المشروع. في سبيل تحقيق أهداف البحث تم الاعتماد على المنهج الوصفي القائم على استقراء وجمع البيانات من الكتب والدراسات والمقالات السابقة المرتبطة بموضوع البحث الحالي. توصلت الدراسة البحثية إلى أنَّ النماذج والأطر التي اقترحها الباحثين لتوضيح علاقة إدارة المعرفة بإدارة المشاريع أكدت على أهمية توظيف عمليات إدارة المعرفة في المجالات المعرفية العشرة 10 لإدارة المشاريع وفي قدرتا على تعزيز فرص نجاح المشروع من خلال اتمام المشروع ضمن التكلفة المناسبة والوقت المناسب وضمن الجودة المطلوبة ومن خلال تعزيز التواصل بين اصحاب المصلحة والأفراد المعنيين ونشر المعرفة بينهم. كما أكدت النتائج على وجود علاقة قوية بين إدارة المعرفة وإدارة المشاريع وتكاملهما في العديد من الجوانب، الأمر الذي يُعزّز من ضرورة تنفيذ المشاريع على اختلاف أنواعها في ظل إدارة العرفة بمختلف عملياتما. أوصت الدراسة بالحاجة إلى زيادة توعية العاملين في المشاريع بأهمية إدارة المعرفة وتبني عملياتها خلال دورة حياة المشروع Project lifecycle من خلال عقد الورش والممارسات المهنية التدريبية الخاصة بذلك لنقل وتبادل ومشاركة المعرفة، وتثقيف العاملين والمدراء بالوسائل والأساليب والممارسات الفعالة لإدارة المعرفة والتي يُمكن توظيفها في إدارة المشاريع. الكلمات المفتاحية: إدارة المعرفة، إدارة المشاريع، تكامل العمليات، نجاح المشروع.

شهد العالم العديد من التغيرات التي تمكنت من التأثير على مختلف مجالات الحياة، حيث أها أثرت على المؤسسات العامة والخاصة، والمشاريع تُعتبر أحد القطاعات التنموية التي تأُثرت بهذه التغيرات، وبالتالي سعت مختلف الإدارات إلى محاولة تحسين جودة المشاريع وضمان نجاحها، فقد أكَّد عنبر (2016) على أنَّ تحقيق جودة المشرعات يُسهم بالضرورة في خفض تكاليف الإنتاج وفي ضمان 


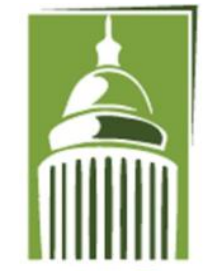

ARF
Global Proceedings Repository American Research Foundation

ISSN 2476-017X

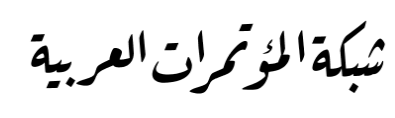

http://arab.kmshare.net/

Available online at http://proceedings.sriweb.org

رضا المستهلك عن الخدمات المقدمة، كما أنه يمنح الثقة للجهة المسؤولة عن المشروع ويُحسيّن من مستواها التنافسي. ولقد أشار دهليز وعنبر (2017) إلى أنَّ ضمان نجاح المشاريع واستمرارها يستلزم بالضرورة وجود معايير وأدوات مناسبة تُقدِّمِ المساعدة المطلوبة لمدراء المشاريع في كيفية إدارة المشاريع وتخطيطها وتنظيمها، حيث تُعتبر المجالات المعرفية لإدارة المشاريع الصادرة من المعهد الأمريكي لإدارة المشاريع (PMAI) من أبرز هذه المعايير، فقد أكَّد العامري (2007) أنَّ للمجالات المعرفية لإدارة المشاريع أهمية كبيرة في المساعدة على تخطيط وتنظيم المشروع ومتابعته بالطريقة المناسبة، حتى أنَّ هذه المجالات المعرفية أصبحت من أهم المواضيع التي تُسهم في تحقيق الجودة للمشاريع. ولقد أشارت العديد من الأدبيات إلى دور تلك المجالات في تحقيق نجاح المشاريع وتقويم مستوى أدائها. (Abdul Rasid et al., 2014; Chauhan \& Srivastava, 2014; Al-Nady et al, 2016). إن إدارة المشروع تشير في الأساس إلى تطبيق المعرفة المناسبة في كامل تفاصيل ومراحل دورة حياة المشروع باعتبار أن الهدف الأساسي من وجود عمليات ومهارات وأدوات ونظم وتقنيات إدارة المعرفة هي المساهمة بشكل أو بآخر في التأثير بفاعلية على نجاح المشروع. و المؤسسات على اختلاف أشكالها تسعى إلى تحسين الأداء التنظيمي، وخلق منافسة بينها وتققيق المزيد من المبيعات، وزيادة حصتها في السوق، وتحسين الإنتاجية والجودة وخلق المنتجات المبتكرة (Al-Shafai, 2017)، ونتيجةٌ لإدراك المجتمعات لأهمية إدارة المعرفة ودورها الأساسي في تحويل المجتمعات نحو الاقتصاد المعرفي الذي يُرِّزّ بشكل أساسي على الاستثمار في الموارد البشرية والفكرية؛ ازداد اهتمام المنظمات على اختلاف أنواعها وأشكالها بالمعرفة والمعلومات لاعتبارها الوسيلة المناسبة للتكيف مع تحديات العصر ومتطلباته المتزايدة، فقد أكَّد حمود (2010) على أنَّ المعرفة تُعتبر المصدر الأساسي لتحقيق الثروة والتميَّز والإبداع خاصة بعدما شهده العالم من تطور تكنولوجي متسارع وواسع، وأضاف دهليز وعنبر (2017) بأنَّ إدارة المعرفة تُمثل مصدراً رئيسياً لتمكين المنظمات من تخفيض التكاليف ورفع مستوى الانتاجية من خلال توليد الايرادات الجديدة. ومن هنا يتضح لنا ضرورة فهم دور إدارة المعرفة وملماذا اعتبرت حاضرة بقوة في المنظمات وريادة أعمال المشاريع من أجل دعم أهدافها وتكوين ثقافة المنظمة وخلق القيمة المضافة وتعزيز الميزة التنافسية ـ وبالتالي فإنَّ حقل إدارة المعرفة وما يتضمنه من مفهومه العصري 


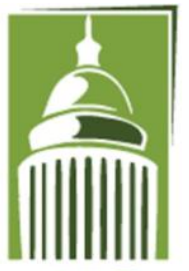

ARF
Global Proceedings Repository American Research Foundation

ISSN 2476-017X

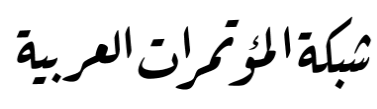

http://arab.kmshare.net/

Available online at http://proceedings.sriweb.org

والتحديات المختلفة التي تواجهه يفرض على بيئة المشاريع التي تُعتبر من أهم البيئات المساهمة في دعم تنمية المجتمعات أن تقوم ببذل جهد أكبر في مجال إدارة المعرفة، حيث أنّ إدارة المعرفة وما تتضمنه من رأس مال فكري تُمثل الأساس الذي ترتكز عليه تقدم الأمم وتطورها، كما أها تمثل الأصول المعرفية غير الملموسة Intangible Assets المؤثرة على إدارة المشروع وتنفيذه. وهذا يُشكِل تحدي كبير بالنسبة لمدراء المشاريع من أجل إحداث نقلة نوعية في إدارة المعرفة وتبني استراتيجياتا وعملياتها في مشاريعهم. وبالتالي تسعى الدراسة البحثية الحالية إلى توضيح أهمية علاقة إدارة المعرفة بإدارة المشاريع من خلال تكامل العمليات ونجاح المشروع.

المشكلة البحثية:

إنَّ التطورات والتغيرات التي شهدها العالم استدعت ضرورة الارتكاز على استراتيجيات داعمة يُمكنن من خلالها التصدي لهذه التحديات، ولكن الخطورة تكمن في انعكاس هذه التحديات على أداء المنظمات، وفي كوفا تستلزم ضرورة الاستجابة السريعة والتمكن من أخذ زمام المبادرة من أجل المحافظة على ديمومتها واستمراريتها.

وعلى الرغم من اعتبار إدارة المعرفة أحد أبرز الحلول التي يُمكن من خلالها تحسين مستوى الأداء وتطويره؛ إلا أنَّ ليرني وريبيري (Lierni and Ribiere, 2008) إدارة المعرفة في تحسين مستوى الأداء وخاصة في إدارة المشروعات، ولكنَّهما أكَّدا من ناحية أخرى على أنه في بيئات المشروع تكتسب المعرفة أهمية كبيرة، حيث يمكن لمدراء المشاريع الاستفادة بشدة من تبادل وترميز المعرفة الضمنية المرتبطة بإدارة المشروعات السابقة. وأكَّد بوليانيانوفا (Polyaninova, 2011) على أنه أثناء تنفيذ المشروع يتم إنشاء أشكال مختلفة من المعلومات والخبرات داخل المنظمة، فإذا لم يتم تسجيل هذه المعرفة المتراكمة ومشاركتها بين المشاريع الأخرى ستضيع هذه المعرفة ولن تكون متاحة لمساعدة المشاريع المستقبلية، وبالتالي قد يؤدي ذلك إلى زيادة تكاليف المشاريع المستقبلية، حيث سيتم استخدام الموارد 


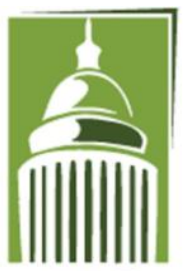

ARF
Global Proceedings Repository American Research Foundation

ISSN 2476-017X

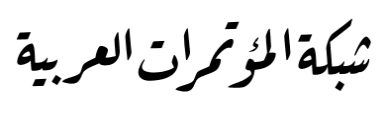

http://arab.kmshare.net/

Available online at http://proceedings.sriweb.org

والوقت والمال المتاح في إعادة تعريف المعرفة التي كانت موجودة في الشركة، وفي حال عدم الحصول على هذه المعرفة وإعادة نشرها تتأثر جودة نتائج المشروع بشكل سلبي.

ومن خلال الرجوع للعديد من الدراسات السابقة سواء العربية منها أو الأجنبية يتبيَّن مستوى الاهتمام بإدارة المعرفة باعتبارها منهجاً حديثاً ومبتكراً لإدارة المنظمات ولأثرها المباشر على تحسين مستوى أداء العمل والانتاجية والخدمات، ولكن وعلى الرغم من الإهتمام بموضوع إدارة المعرفة وتطبيقها في مختلف المجالات؛ إلا أنَّ الدراسات النظرية والتطبيقية وخاصة العربية منها والتي تناولت طبيعة العلاقة التكاملية بين إدارة المعرفة وإدارة المشاريع تُعتبر محدودة للغاية، كما أنه وعلى حد علم الباحث لا توجد دراسة عربية تبحث في هذا المجال بالتحديد بشكل متكامل، فمعظم الدراسات العربية تناولت المفهومين (إدارة المشاريع وإدارة المعرفة) بشكل منفصل ودرست علاقتهما و تأثيرهما بعوامل أخرى مثل الميزة التنافسية ومستوى الأداء وتحسين الجودة. ونظراً لأهمية بيئة المشاريع وما تتضمنه من عناصر بشرية وتقنية عديدة وبما تُسهم به في تحقيق عجلة التنمية الاقتصادية؛ تتمثل مشكلة الدراسة الحالية في توضيح أهمية علاقة إدارة المعرفة بإدارة المشاريع من خلال تكامل العمليات ونجاح المشروع. ومن هنا يُمكن تلخيص مشكلة الدراسة في السؤال التالي: كيف تُسهم إدارة المعرفة في تحقيق تكامل عمليات ونجاح إدارة المشاريع؟

في سبيل تحقيق أهداف الدراسة سيتم الاعتماد على المنهج الوصفي القائم على استقراء وجمع البيانات من الكتب والدراسات والمقالات السابقة المرتبطة بموضوع البحث الحالي. يُعتبر المنهج الوصفي أحد الأشكال القائمة على وصف ظاهرة أو موضوع معين، وتصويرها نوعياً من خلال جمع البيانات والمعلومات المرتبطة بالظاهرة وتحليلها وتفسيرها ضمن الأسس العلمية الصحيحة (عدس، 


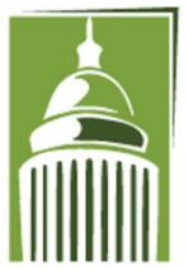

ARF
Global Proceedings Repository American Research Foundation

ISSN 2476-017X

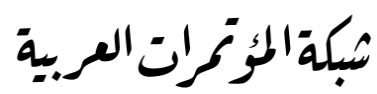

http://arab.kmshare.net/

Available online at http://proceedings.sriweb.org

ما يُميّز الدراسة البحثية الحالية عن غيرها من الدراسات والأبحاث السابقة هي أها تبحث في بجال حيوي وغاية في الأهمية وهو أهمية العلاقة بين إدارة المعرفة وإدارة المشاريع والأثر المختمل من عمليات إدارة المعرفة على المجالات المعرفية لإدارة المشاريع، لذلك تُعتبر الدراسة الحالية مكملة للنقص الموجود في الدراسات العربية السابقة بشكل خاص. ويُمكن تحديد أهمية الدراسة من خلال النقاط

1. تُعتبر الدراسة الحالية محاولة لإبراز أهمية إدارة المعرفة وذلك من خلال توظيف عمليات المعرفة في تحسين أداء المشاريع

$$
\text { وتقليل فرص فشلها وزيادة فرص نجاحها. }
$$

2. تُعتبر الدراسة الحالية من الدراسات القليلة التي تناولت موضوع أهمية إدارة المعرفة في إدارة المشاريع، مما يجعل البحث الحالي مصدراً جديداً في هذا المجال يُمكن دعمه والتأكيد على نتائجه من خلال اجراء المزيد من الدراسات والبحوث المستقبلية.

1. ترتبط الدراسة الحالية بعمل وأداء المشاريع على اختلاف أنواعها والتي تُعتبر محرك أساسي ورئيسي في دعم عجلة التقدم

$$
\text { الاقتصادي في الدول. }
$$

2. تسعى الدراسة الحالية إلى تزويد مدراء المشاريع بالآليات والطرق التي يُمكن من خلالما تنفيذ المشاريع بنجاح وعلى مستوى عالي من التميز من خلال توضيح أهمية توظيف ودمج عميلات إدارة المعرفة مع المجالات المعرفية لإدارة المشاريع. 3. تسعى الدراسة الحالية إلى الارتقاء بمستوى المشاريع محليا وعربيا من خلال الاستغلال الأمثل للمعرفة (توليد المعرفة وتزينها ومشاركتها وتطبيقها) وكيفية دمجها مع المجالات المعرفية لإدارة المشاريع من أجل تحسين جودة المشاريع على اختلاف 


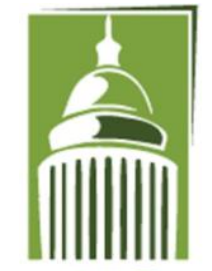

ARF
Global Proceedings Repository American Research Foundation

ISSN 2476-017X

Available online at http://proceedings.sriweb.org

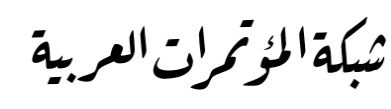

http://arab.kmshare.net/

\section{مفهوم أصول المعرفة وإدارة المعرفة}

لقد اكتسب مفهوم المعرفة أهمية خاصة من قبل الباحثين، حيث أكَّد أبو فارة (2004) على أنَّ المعرفة تُمثل حقل معرفي ناتج بشكل أساسي من النشاط والعقل البشري، فقد بدأ الانسان السعي لتحقيق المعرفة من أجل الخحافظة على حياته ومواجهة مختلف التحديات والصعوبات التي تواجهه، وانطلاقاً من هنا تعددت محاولات الباحثين في وضع مفهوم واضح ومحدد للمعرفة، إلا أنّ هذه الخحاولات لم ينتج عنها إعطاء المعرفة مفهوما شاملا، ولكن اتفق الباحثين على أنَّ المعرفة تُمثل بالنسبة للأفراد والشعوب ثروة حقيقية ومورد أساسي لتعزيز المستوى التنافسي (صويص، 2011). فقد عرَّها العلواني (2001) بأها تفاعل يجري بين المعرفة الضمنية التي تتمثل بخبرات الأفراء ومهاراقم المتنوعة والمعرفة الظاهرة التي تنتج من خلال التفاعل مع البيئة الخارجية، وعرَّها رزوقي (2008) بأغما رأس مال فكري إذا تم استثمارها واستغلالما بالشكل الصحيح من قبل المنظمة، وأشار التلباني وآخرون (2015) إلى وجود علاقة متبادلة بين المعلومات والمعرفة والفعل، حيث تُعبِّر المعرفة عن المعلومات القادرة على دعم الفعل وتويلها إلى عمل، وبالتالي أكَّد مافودزا و نجولوب (Mavodza and Ngulube, 2012) على أنَّ المعرفة تُمثل ما يحتاجه الأفراد من معلومات ومعارف من أجل القيام بأعمالمم بالشكل المطلوب.

وقد جادل ليفين (Levin, 2011) بأنه لا يوجد تعريف معياري معتمد لأصول المعرفة ، لكن يُنظر إيلها على أخها خبرة بشرية مقننة وخزنة في نسق رقمي، وتستخدم لخلق قيمة تنظيمية. ينصب التركيز على استخدام أصول المعرفة لتعزيز التفاهم وتوفير التوجيه لاتخاذ القرار وتسجيل الحقائق حول القرارات المامة. ويكن لأصول المعرفة، إذا توفرت في نسق يسهل الوصول إليها والاعتماد عليها ، أن تسمح بالعمل على المشروعات بإشراف أقل وأن يتم إعدادها بحيث يككن للأشخاص الوصول إليها في أي وقت. كما أشار إلى أن عملية إنشاء أصول المعرفة تنطوي على تحديد العمل والأهداف المهمة للمنظمة، وتقييم المجالات التي قد تكون فيها المنظمة ضعيفة، وتحديد كيفية حدوث أي خسارة للخبرة، وتقييم الحواجز والعقبات في المنظمة. ونظرًا لأن الكثير من أصول المعرفة المتوفرة لم يتم توثيقها، أو أغها مازالت ضمنية، فإنه يمكن فقداخا بسهولة خاصةً عندما يغادر الموظفين الخبراء المؤسسة أو المشروع لأي سبب 


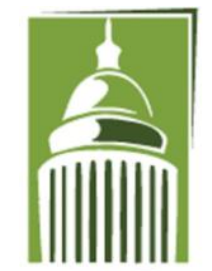

ARF

\section{Global Proceedings Repository}

\section{American Research Foundation}

ISSN 2476-017X

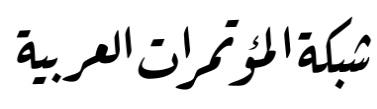

http://arab.kmshare.net/

Available online at http://proceedings.sriweb.org

كان ـ لذا يجب الاهتمام بالحصول على الأصول المعرفية عبر إجراء المقابلات التي تركز في كثير من الأحيان على استخلاص لب المعرفة الضمنية من أذهان وعقول الخبراء، أو القيام بالملاحظات أثناء العمل من قبل شخص يتمتع بالخبرة. كما أن الوثائق أيضا إلى جانب السياسات والإجراءات والمبادئ التوجيهية هي أصول معرفية يمكن جمعها وإتاحتها بسهولة.

أصول المعرفة كما بينها ليفين (Levin, 2011) تشير إلى الموارد الفكرية المتراكمة للمنظمة. إها المعرفة التي تمتلكها المنظمة في مشروع ما، وهي قوتا العاملة في شكل معلومات وأفكار وتعلم وفهم وذاكرة تنظيمية ورؤى ومهارات معرفية وفنية وقدرات متخصصة .وهذا يعني أن القوى العاملة في المشروع وقواعد البيانات والمستندات والأدلة والسياسات والإجراءات والبرجيات وبراءات الاختراع هي مستودعات لأصول المعرفة الخاصة بالمنظمة أو المشروع. فهي تكون وتنتج ليس فقط من قِبل منظمة بل أيضًا من العملاء والموردين والشركاء والمساهمين وأصحاب المصلحة والأطراف المعنية باختلاف تصنيفاقم ومستوياقم وتوجهاتم وتوقعاقم ومستوى تأثيرهم وسلطتهم ونفوذهم. ولذلك ، فإن أصول المعرفة هي "معرفة كيفية" في كل مؤسسة وهي متاحة للاستخدام وللاستثمار والنمو والوفرة .يعد بناء أصول المعرفة وإدارتا مكونًا أساسيًا لأي مؤسسة من أجل خلق القيمة للعملاء للمساعدة في الحفاظ على النجاح الكلي لأداء المؤسسة.

بالإضافة إلى ذلك، توصف محتويات الأصول المعرفية من البيانات والمعلومات بسهولة استخدامها واستردادها من قبل المتخصصين في المشروع .ومع ذلك ، فإن إنشائها يستغرق وقتًا لضمان حصول كل شخص على فهم شامل وواسع ومشترك لمعنى البيانات الوصفية المختلفة التي سيتم استخدامها من أجل أن تكون قاعدة معرفية معلوماتية عن البرنامج أو المشروع. وبالتالي ، فإن إدارة المعرفة هي عملية تعتمد على الطريقة المثلى التي يمكن لأصحاب المصلحة من خلالها دمج أصولمم المعرفية الفردية، وكلما كان هناك نجاح في دمج للأصول المعرفية فإن ذلك يؤدي إلى برامج ومشاريع ناجحة. Levin, 2011) 


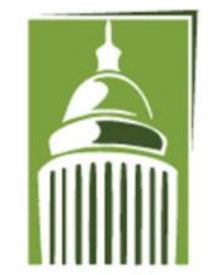

ARF Global Proceedings Repository
American Research Foundation

ISSN 2476-017X

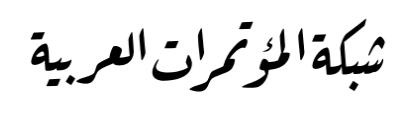

http://arab.kmshare.net/

Available online at http://proceedings.sriweb.org

ولقد ساهم تزايد إدراك أهمية المعرفة بإثارة فضول الباحثين ورغبتهم في دراستها ومحاولة التوصل إلى العمليات التي يُكن من خلالها إدارتا بفاعلية، وتوصلوا من خلال ذلك إلى أنّ إدارة المعرفة هي الخاصية التي تسعى المنظمات إلى تحقيقها واستغلالها في الوقت الحاصر والمستقبل (Mas-Machuca, 2014)، ومن هنا بدأت إدارة المعرفة تتخذ مكانة كبيرة في عالم ريادة الأعمال والمشاريع باعتبارها تُسهم بشكل كبير وملحوظ في تنمية إدراك المنظمات بأنَّ وجود المعرفة ليس له قيمة دون العمل على إدارة هذه المعرفة بالأسلوب المناسب، حيث أنَّ المعرفة بطبيعتها الغالبة تُعتبر ضمنية، وبالتالي تحتاج إلى الكشف عنها وتوليدها وانتاجها وتوزيعها ومشاركتها وتطبيقها بشكل فعال.

لقد نالت إدارة المعرفة أهمية واسعة وكبيرة ظهرت من خلال الدراسات المتخصصة في هذا المجال ووجهات نظر الباحثين في تعريف مفهوم إدارة المعرفة، فهنالك بعض المتخصصين اعتبروه مصطلح تقني، وآخرون نظروا إليه باعتباره مفهوم غير ملموس، والبعض الآخر اعتبر إدارة المعرفة تُشير إلى الثقافة التنظيمية وتطوير المعلومات (صويص، 2011). وقد عبّر الكبيسي (2005) عن مفهوم إدارة المعرفة من خلال تناول العديد من المجالات، فقد أشار إليه كمصطلح يتضمن العمليات والأدوات والسلوكيات التي يتولى تنظيمها العاملين في المنظمة، حيث يقومون بتوليدها وتوزيعها ونقلها وتطبيقها وتخزينها بصورة تُحكِِّن المنظمة من تحقيق ميزة تنافسية طويلة الأمد.

وعرَّفها بدر (2010) بأها العملية التي تتضمن تحويل المعرفة وتخزينها وتطبيقها واسترجاعها للاستفادة منها في انتاج خدمات مبتكرة واستخدام مخرجامَا في عميلات التعلم من أجل تحقيق رسالة المنظمة. كما أشار تقرير هيئة الأمم المتحدة (2004) إلى أنَّ إدارة المعرفة تتضمن إدراك قيمة وأهمية الممتلكات الموجودة في المنظمة، والتركيز على استغلال هذه الموارد بالشكل المناسب بصورة تُساعد المنظمة على التغلب على العوائق والمشكلات والتحديات التي تواجهها، وتُمَكِنها كذلك من تحسين قدرقا على اتخاذ القرارات وتطوير جودة المنتجات والسلع والخدمات. 


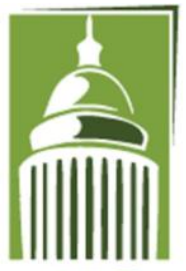

ARF
Global Proceedings Repository American Research Foundation

ISSN 2476-017X

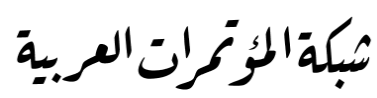

http://arab.kmshare.net/

Available online at http://proceedings.sriweb.org

ومن الضروري الالتفات إلى أنَّ إدارة المعرفة لا تعمل في فراغ، وإنما تتطلب وجود بيئة تنظيمية مناسبة لذلك وتتوافر فيها العناصر والأدوات اللازمة، فقد أكد المودالال (AlModallal, 2012) على أنَّ طبيعة البيئة التنظيمية في المؤسسة يُككن أن تدعم عملية إدارة المعرفة وتُسهم في تحقيق الفاعلية المطلوبة، ويُمكن من ناحية أخرى أن تُعوّق قدرة المؤسسة على تحقيق أهدافها، كما بيَّن العلواني (2001) وأخافان وآخرون (Akhavan et al., 2010) أنَّ إدارة المعرفة تتطلب بشكل أساسي التركيز على أربعة عuek, ) Dناصر أساسية، هي الثقافة التنيمية، والهيكل التنظيمي، وتكنولوجيا المعلومات، والقيادة التنظيمية. وأكَّد دويك 2001) على وجود ثلاث متطلبات أساسية لإدارة المعرفة، هي المتطلب التكنولوجي، والمتطلب التنظيمي واللوجستي، والمتطلب الاجتماعي.

وبالنظر إلى ما سبق يُمكن اعتبار إدارة المعرفة كوسيلة وآلية يُكن من خلالها استغلال مهارات وخبرات الأفراد ونقل المعرفة المتولدة بينهم بصورة تُعزز العمل الجماعي والمتكامل للبحث عن المعلومات التي من شأها أن تخدم قدرة المنظمة على تقعيق أهدافها وإدراك تطلعاتما.

جادل ليفين (Levin, 2011) حول ضرورة إبراز أهمية إدارة المعرفة (KM) في المشاريع، وأكد أن المشكلة الرئيسية تتمثل في عدم وجود تعريف موحد لـ KM بخلاف إدارة المشاريع وإدارة البرامج وإدارة المحافظ وإدارة المخاطر وإدارة المشتريات وغيرها من الإدارات وفق ما يصدر من PMI. هذا يعني أنه حتى يتم تطوير معيار موحد ومقنن KM في المشروع، يجب على كل منظمة مراجعة التعريفات المتاحة واختيار واحدا يناسب أهداف المنظمة. يجب أن يركز على الأفراد والحكم ليس فقط على الأدوات 


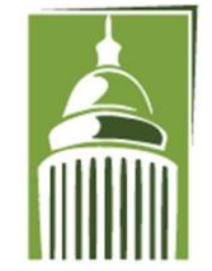

AR F Global Proceedings Repository
American Research Foundation

ISSN 2476-017X

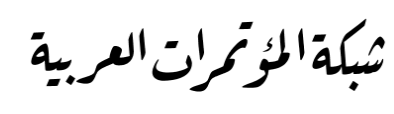

http://arab.kmshare.net/

Available online at http://proceedings.sriweb.org

والتقنيات. كما تتطلب إدارة المعرفة أهدافها الخاصة ومقاييسها لإظهار تقدمها في الوفاء بتلك الأهداف لتكون قادرة على إثبات فوائدها وليس فقط إظهار تكاليفها .في حين يؤكد المنظور العام في هذه الدراسة البحثية على أهمية الأشخاص للعملية، يجب النظر إلى مواقف الأفراد المشاركين، من المساهمين الفرديين والمستخدمين إلى التنفيذيين، بالإضافة إلى الثقافة التنظيمية القائمة بتحهها. كما بين ليفين بأنه لابد من جعل إدارة المعرفة حزمة عمل في هيكل تصنيف المشروع لتسهيل ضرورة تعزيز إدارة المعرفة على برنامج أو مشروع، فعندما يتم تطوير هيكل تصنيف العمل في المشروع (WBS) ، يجب وضع عنصر منفصل جانباً لمركز KM بنفس طريقة إدارة البرنامج أو المشروع · ويمكن أن يتحلل هذا العنصر بعد ذلك إلى عناصر فرعية، مثل الإنشاء والمراجعة والنشر والموافقة والاستخدام والتقييم، بحيث يمكن تعيينه لأفراد محددين كحزم برنامج أو حزم عمل في البرامج. على سبيل المثال ، قد يكون من المناسب تعيين عنصر KM إلى موظفي مكتب إدارة البرنامج الذين يدعمون البرنامج · إن إدراج KM في WBS سوف يسهل التواصل حوله مع أصحاب المصلحة في البرنامج أو المشروع، وسيمكن الموارد من تعيينها، وسيضع مناهجًا محددة لرصد التقدم المحرز والتحكم فيه. ولابد أيضا من إنشاء نقطة اتصال لـ KM على كل برنامج ومشروع ويعمل مع شخص ما في مكتب إدارة برنامج المنظمة. فعلى الرغم من أن العديد من المنظمات قد أنشأت منصب كبير موظفي المعرفة (CKO) وقد يكون لديها مكاتب إدارة المعرفة بسبب أهمية البرامج والمشروعات للأهداف العامة والأهداف الاستراتيجية للمؤسسة وإدارة العديد من المنظمات من خلال المشاريع. لذا فإنه يمكن ربط دورة حياة KM مع دورة حياة البرنامج أو إدارة المشاريع واستخدام لوحة الحوكمة في مراجعات بوابة المرحلة الرئيسية وطرح الأسئلة حول استخدام أصول المعرفة وخلقها في إدارة المشروع. وتُشير الأدبيات إلى وجود العديد من الفوائد لإدارة المعرفة، حيث أشار نجم (2004) وصويص وآخرون (2011) إلى مجموعة من هذه الفوائد والتي تتضمن: 1. تُعتبر إدارة المعرفة وسيلة أساسية لرفع جودة المنتجات وتحسين مستوى الانتاجية. 
Global Proceedings Repository

\section{American Research Foundation}

ISSN 2476-017X

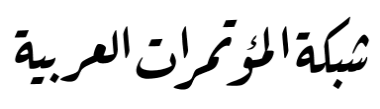

http://arab.kmshare.net/

Available online at http://proceedings.sriweb.org

2. تُعزّز إدارة المعرفة من قدرة المنظمات على تحسين الأداء التنظيمي.

3. تُعد إدارة المعرفة وسيلة فعالة لاستثمار رأس المال الفكري واستغلاله بالشكل المناسب.

4. تُُثل إدارة المعرفة عملية متكاملة قادرة على تنسيق أنشطة المنظمة.

5. تُسهم إدارة المعرفة في تعزيز قدرة المنظمات على تحقيق الميزة التنافسية المطلوبة.

6. تُعزّز إدارة المعرفة من تمكين المنظمات من مواجهة التغيرات والتحولات السريعة.

وبصورة عامة تحدف إدارة المعرفة إلى تحقيق ما يلي (رزوقي، 2008؛ صويص وآخرون، 2011):

1 1. مواجهة التحديات التي تواجه المنظمات المعاصرة.

2. وضع الحلول المناسبة للمشكلات التي تواجه المنظمات.

3. تشجيع الأفراد على المشاركة المعرفية والمساهمة في تحسين مستوى المنظمة.

4. خلق بيئة تنظيمية فعالة ومحفِّة.

5. تحويل المنظمات من الاقتصاد التقليدي إلى الاقتصاد المعرفي.

6. تطوير أداء العاملين وزيادة مستوى الانتاج.

7. خلق قيمة مضافة للمنتجات والخدمات.

\section{عمليات إدارة المعرفة}

لقد نجم عن المفاهيم المتعددة والمتنوعة التي قُِّدمت للمعرفة محاولات عديدة للباحثين من أجل تقديم نماذج لإدارة المعرفة، مثل أنموذج البنك الكندي التجاري لإدارة المعرفة (Canadian Imperial Bank of Commerce) والمبني بشكل أساسي على مفهوم الثقافة المعرفية ونشر قيم المعرفة، ويتكون هذا النموذج بشكل أساسي من أربعة عناصر أساسية تُساعد المنظمة على اتخاذ الاجراءات المناسبة التي يُككن من خلالها إدارة المعرفة بنجاح، وتشمل على التعلم الفردي من خلال منح الأفراد مسؤولية التعلم 


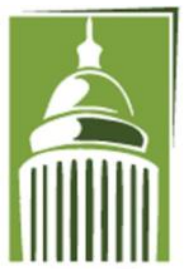

ARF
Global Proceedings Repository American Research Foundation

ISSN 2476-017X

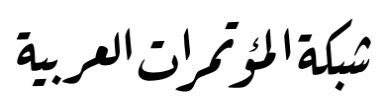

http://arab.kmshare.net/

Available online at http://proceedings.sriweb.org

المستمر بالاعتماد على أنفسهم، وتعلم الفريق من خلال تحميل المجموعات مسؤولية التعلم الذاتي، وتعلم المنظمة من خلال العمل على نشر المعرفة في المنظمة، وتعلم العميل (Darling, 1996). كما قدَّم بورديو وكولارد ( (Coullard, 1999 نموذجاً لإدارة المعرفة يشمل على ثماني خطوات أساسية لتطبيق إدارة المعرفة بنجاح، والتي تتضمن غَيئة الفريق، وفهم أهداف الأعمال والرؤية الاستراتيجية للمؤسسة، وتحديد عمليات الأعمال، وتحديد التطبيقات ومتطلبات الاستخدام، وتصميم البنية المعرفية والبيانات، وتصميم البنية الفنية، ومرحلة التنفيذ، ومرحلة التعلم والتحسين والتي تشمل تقييم مستوى الجهود وتسعى إلى تحسين العمليات والأدوات وإجراء التعديلات المناسبة.

وقام دوفي (Duffy, 2000) بتقديم نموذج أخر لإدارة المعرفة مرتكزاً بشكل أساسي من منطلق أنَّ المنظمة تحصل على البيانات والمعلومات من البيئة الخارجية المحيطة فيها، وعندما تتشارك جهود الأفراد والعمليات يتم تحويل هذه المعلومات إلى معرفة تؤدي في النهاية إلى انتاج وتوليد السلع والخدمات، مما يؤثر على مستوى الأداء المؤسسي ويُسهم بفاعلية بزيادة ثروة المنظمة. ويُشير دوفي في نموذجه إلى أنَّ إدارة المعرفة هي عملية يتم من خلالما تحقيق المعرفة الضمنية والصريحة، وتوليد العائدات التي (Duffy, 2000) ترتكز على العنصر البشري باعتباره عنصر أساسي في تحقيق النتائج المطلوبة. ويتضمن هذا النموذج على عملية إدارة المعرفة من

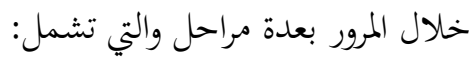

\footnotetext{
1 1. الحصول على المعرفة؛ والتي تتضمن الشراء والتوليد. 2. تنظيم المعرفة؛ والتي تتضمن التصنيف والتبويب والرسم.

3. استرجاع المعرفة؛ والتي تتضمن البحث والوصول. 4. توزيع المعرفة؛ والتي تتضمن المشاركة والنقل. 5. إدامة المعرفة؛ وتشمل التنقيح والنمو والتغذية.
} 


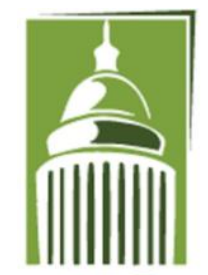

ARF
Global Proceedings Repository American Research Foundation

ISSN 2476-017X

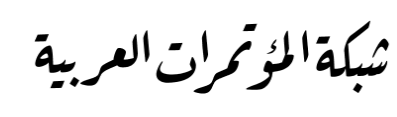

http://arab.kmshare.net/

Available online at http://proceedings.sriweb.org

والشكل التالي يُشير إلى نموذج عمليات إدارة المعرفة وفقاً لنموذج دوفي (Duffy, 2000).

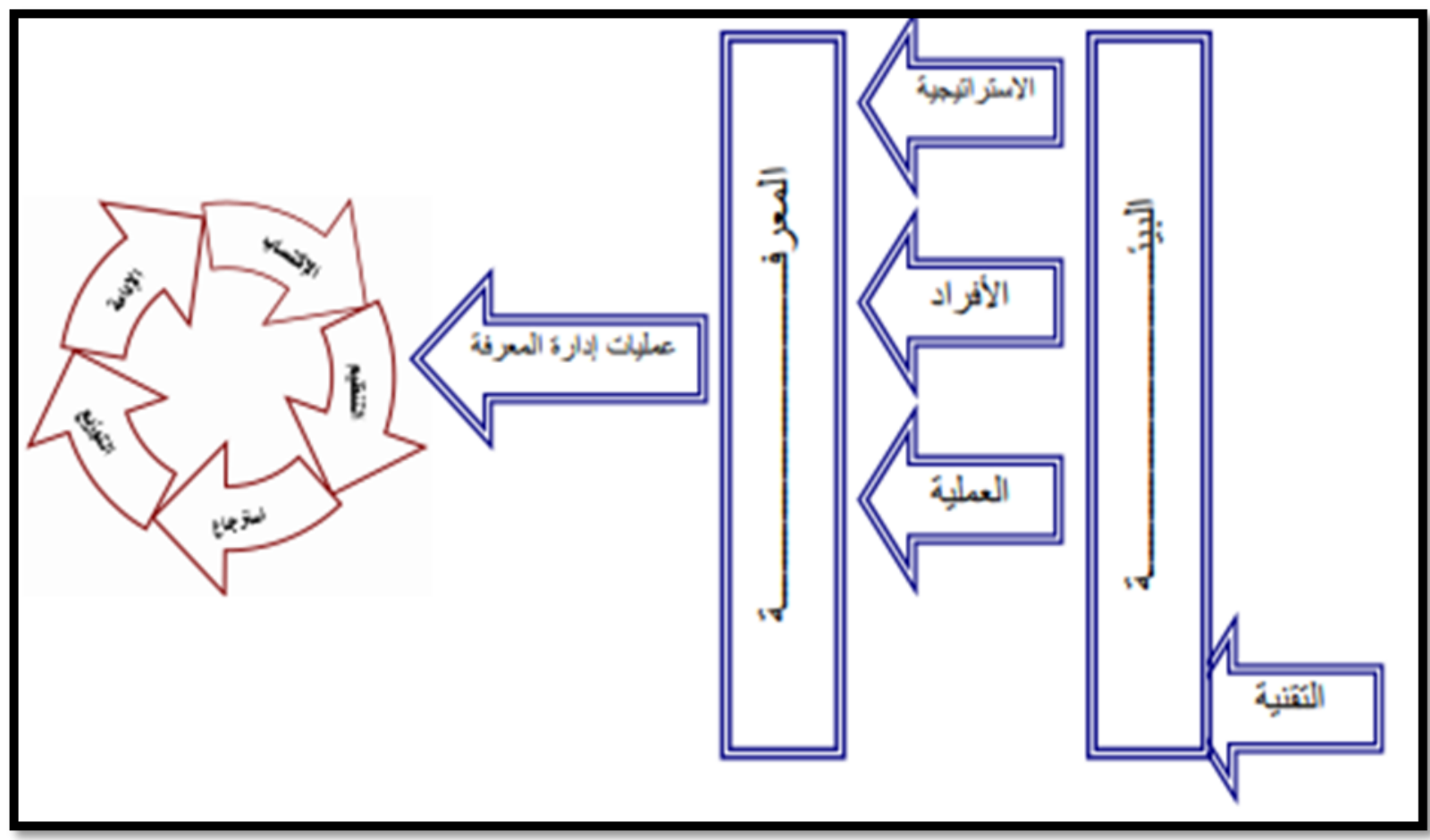

الشكل التوضيحي -1: نموذج عمليات إدارة المعرفة وفقاً لنموذج دوفي (Duffy, 2000)

وعند التمعن بالمفاهيم التي قُدِّمت لإدارة المعرفة يُمكن ملاحظة أها ركزت جميعها على كون إدارة المعرفة تُمثل عملية، وهذا يعني أنّ الحصول على المعلومات من المصادر الداخلية والخارجية لا يُمكن الاستفادة منها دون وجود عمليات تُمُكِن من الوصول إليها وتخزينها واسترجاعها عند الحاجة إليها، وبالتالي فقد أكَّد صويص وآخرون (2011) على أنَّ المعرفة تُعتبر مجردة القيمة إذا ما تمت عملية تشخيصها وتوليدها وتخزينها واسترجاعها، حيث أنَّ هذه العمليات تُمكِِن من جعل المعرفة قابلة للتطبيق في مختلف الميادين، حيث أُطلق على هذه العمليات مفهوم "عمليات إدارة المعرفة"، حيث بيَّن حجازي (2005) أنَّ هذه العمليات تمتاز بأها متتابعة ومتكاملة وبالتالي تعتمد على بعضها البعض، وكل عملية ترتكز على العملية التي تسبقها وتدعم العملية التي تليها. وبشكل عام تتضمن عمليات إدارة المعرفة ما يلي (الكبيسي، 2005؛ صويص وآخرون، 2011): 


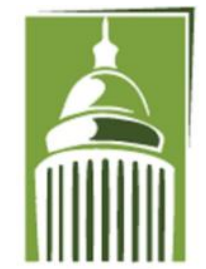

ARF

\section{Global Proceedings Repository \\ American Research Foundation}

ISSN 2476-017X

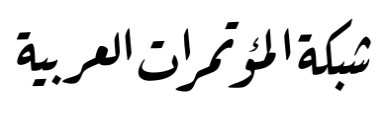

http://arab.kmshare.net/

Available online at http://proceedings.sriweb.org

1. تشخيص المعرفة Knowledge Diagnose: وهي التي يتم من خلالها تحديد نوع المعرفة الموجودة، كما يتم مقارنتها بغيرها من أنواع المعرفة وتحديد الفجوة. وأشار نايف (2007) إلى أنَّ عملية التشخيص تُعتبر مهمة لأهما تُسهم في تحديد نوع معرفة المنظمة وامكانية التعامل معها، بالإضافة إلى تحديد الأشخاص المناسبين لهذا التعامل في المنظمة وتحديد مهامهم وواجباقم. كما جادل التلباني وآخرون (2015) بأنّ نجاح عملية إدارة المعرفة يتوقف على نجاح عملية التشخيص ومستوى دقتها، حيث أنَّ عملية التشخيص تُحِّدِد طبيعة المعرفة المناسبة وتُمُكِّن الأفراد من وضع الحلول المناسبة للمشكلة، كما أفا تعتمد بصورة أساسية على استخدام الأدوات والوسائل البحثية المناسبة لذلك. 2. توليد المعرفة Knowledge Creation: توليد المعرفة يعني تضافر جهود أفراد العمل ومشاركتهم مع بعضهم البعض من أجل إنتاج رأس مال معريف يُمكن من خلاله تحديد المشكلات وإيباد الحلول المبتكرة ها، كما أها تُمثل الأنشطة التي تسعى المنظمة إلى تطبيقها من أجل الحصول على المعرفة المناسبة، سواء المعرفة الضمنية أو المعرفة الصريحة. ويُعتبر نموذج دورة إدارة المعرفة (SECI) من أبرز النماذج المستخدمة في عملية توليد المعرفة وايجادها بالشكل المناسبة في المنظمة، بحيث يتكون هذا النموذج من أربعة عمليات أساسية، تتضمن (Survay, 2005): أ. التفاعل الاجتماعي (Socialization): وهي المعرفة التي يتم انتاجها عندما يقوم الأفراد بالتشارك بالمعرفة الضمنية.

ب. التجسيد (Externalization): وهي التي تتضمن تحويل المعرفة الضمنية إلى معرفة صريحة وواضحة. ت. التجميع (Combination): وهي التي تتولد نتيجةً للاتصال بين الأفراد والجماعات، ويتم من خلالها تحويل المعرفة الصريحة الواضحة إلى معرفة أكثر وضوحاً. ث. التذويب أو الاستيعاب (Internalization): وهي التي تتضمن تحويل المعرفة الصريحة إلى معرفة ضمنية، مثل عملية تحويل معرفة المؤسسة التنظيمية إلى روتين يومي والمبادرات استراتيجية. الشكل التالي يُبيِّن كموذج دورة إدارة المعرفة (SECI). 


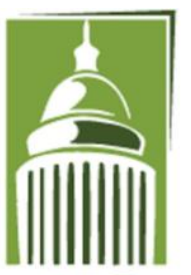

ARF

\section{Global Proceedings Repository \\ American Research Foundation}

ISSN 2476-017X

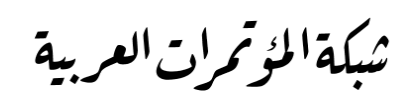

http://arab.kmshare.net/

Available online at http://proceedings.sriweb.org

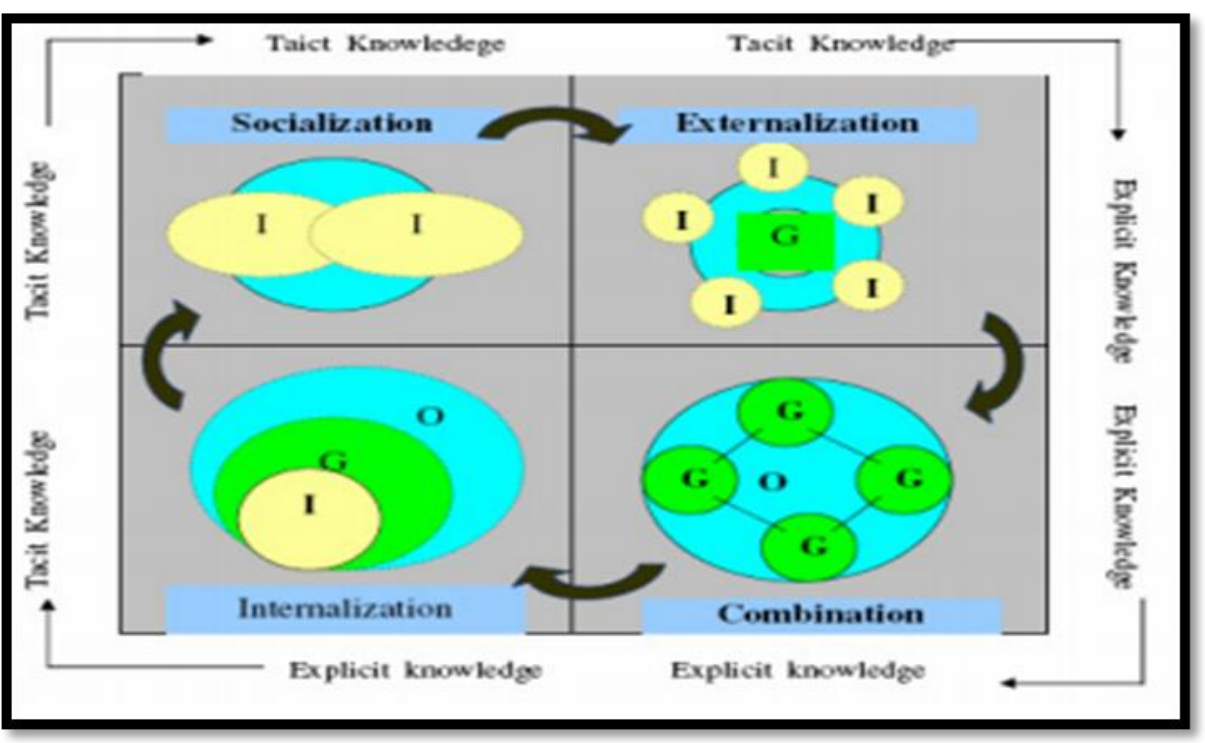

(Nonaka and Konno, 1998) (SECI) الشكل التوضيحي- 2: نموذج دورة إدارة المعوفة (تحويل المعرفة)

3. خزن المعرفة Knowledge Storing: وهي العملية التي تتضمن الاحتفاظ بالمعرفة وامكانية استرجاعها

وإدامتها وتنظيمها وتسهيل عملية البحث عنها، وتُمثل هذه العملية بالنسبة للمؤسسة الذاكرة التنظيمية التي تحتوي على

أهم وأبرز المعلومات المعرفية المرتبطة بها، وقد أشارت دروزة (2008) إلى أنَّ المنظمات تواجه في العديد من الحالات

مخاطر كبيرة نتيجةً لفقداهما المعرفة الخاصة بها، وهذا نتيجةً لمغادرة بعض الأفراد المنظمة لأسباب عديدة، الأمر الذي حتَّم على المؤسسات ضرورة الاحتفاظ بالمعرفة من أجل تسهيل الوصول إليها وقت الحاجة.

4. نقل المعرفة ومشاركتها Knowledge transfer and Sharing: وتتضمن عملية توزيع المعرفة بين مختلف أفراد المنظمة، حيث يتم توزيع المعرفة الضمنية من خلال التواصل والحوار بين الأفراد، أما المعرفة الصريحة فيتم توزيعها من خلال الوثائق وتعميم النشرات الداخلية بين الموظفين. 


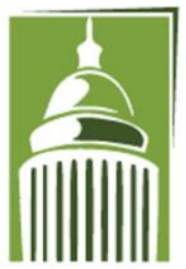

ARF
Global Proceedings Repository

American Research Foundation

ISSN 2476-017X

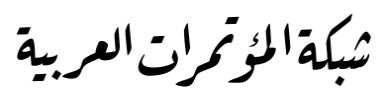

http://arab.kmshare.net/

Available online at http://proceedings.sriweb.org

5. تطبيق المعرفة Knowledge Application: وهي الهدف الأساسي من عملية إدارة المعرفة، وتتضمن

استغلال المعرفة في الوقت المناسب واستثمارها بالطرق الصحيحة من أجل مواجهة المشكلات والتحديات التي تواجه

المنظمة، بحيث يكون الغرض الأساسي منها التطبيق هو تحقيق غايات المنظمة وأهدافها الاستراتيجية.

مفهوم المشروع وإدارة المشروع

المشروع هو عبارة عن مؤسسة مؤقتة يتم إنشاؤها لغرض تقديم منتج أو أكثر من منتجات الأعمال وفقًا لحالة العمل المتفق Pليها (خير الدين، 2012). كما عَّف دليل هيئة إدارة المشاريع للمعرفة ( Project Management Body of The ) المشروع بأنه عملية تمف إلى استحداث منتج أو خدمة أو نتيجة فريدة (Knowledge (PMBOK) Project Management Institute (PMI), 2008 وأشار العلي (2011) إلى أنَّ كل مشروع له بداية وغاية ويهدف بالضرورة إلى اناتج خدمة أو منتج مميز، حيث يتطلب ذلك توافر موارد مختلفة يتم تحديدها بناءً على حجم وضخامة المشروع. وأضافت خالد (Khalid, 2017) أنَّ المشاريع تختلف عن بعضها البعض في حجمها، ودرجة تعقيدها، وأهدافها، ووقت بدايتها وغايتها، ومقدار عدم التأكد بالإضافة إلى احتياجاهما المالية.

أما إدارة المشاريع فتُعَّفَ بأها الطريقة التي يتم من خلالما تطبيق المعارف والمهارات على خختلف أنشطة المشروع من أجل تحقيق مroject Management ( هو متوقع من المشروع (عنبر، 2016). ووفقًا لدليل هيئة إدارة المشاريع للمعرفة (Body of Knowledge (PMBOK) والتقنيات المناسبة يمكن أن يكون له تأثير كبير على نجاح المشروع (PMI, 2008)، حيث أنَّ لمدف الرئيسي من إدارة المشروع هو ضمان إكمال المشروع في النطاق المطلوب المحدد من قبل أصحاب المصلحة، وضمن ميزانية المشروع ، وفي الوقت 


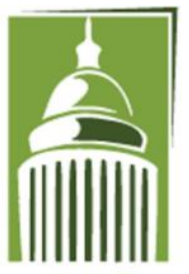

ARF

\section{Global Proceedings Repository}

American Research Foundation

ISSN 2476-017X

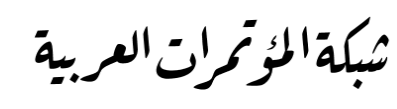

http://arab.kmshare.net/

Available online at http://proceedings.sriweb.org

الخحدد وتقديم منتج أو خدمة عالية الجودة كنتيجة خائية. ويُشير خير الدين (2012) إلى أنَّ إدارة المشروع عُمثل علم وفن في حل المشكلات ضمن الوقت المحدد والتكلفة المحددة والموارد المتوفرة. وبشكل عام تدف إدارة المشاريع إلى تحقيق العديد من الأهداف، منها: 1. - ت تحقيق أهداف المشروع. 2. تحقيق رضا العميل من خلال تقديم خدمات ذات جودة عالية وبتكلفة أقل وخلال وقت سريع. 3. تيادة مستوى الربح.

4. مساعدة مدراء المشاريع على التغلب على المشاكل والتحديات التي تواجهم أثناء تنفيذ المشروع. 5. مساعدة مدراء المشاريع على اتخاذ القرارات المناسبة التي تُسهم في تحقيق أهداف المشروع بفاعلية.

أما العلي (2011) فقد أشار إلى وجود أربعة أهداف عامة للمشروع، والتي تتضمن تقديم الخدمة، والربح، والأهداف الاجتماعية وتحقيق النمو. يوضح الشكل التالي هذه الأهداف.

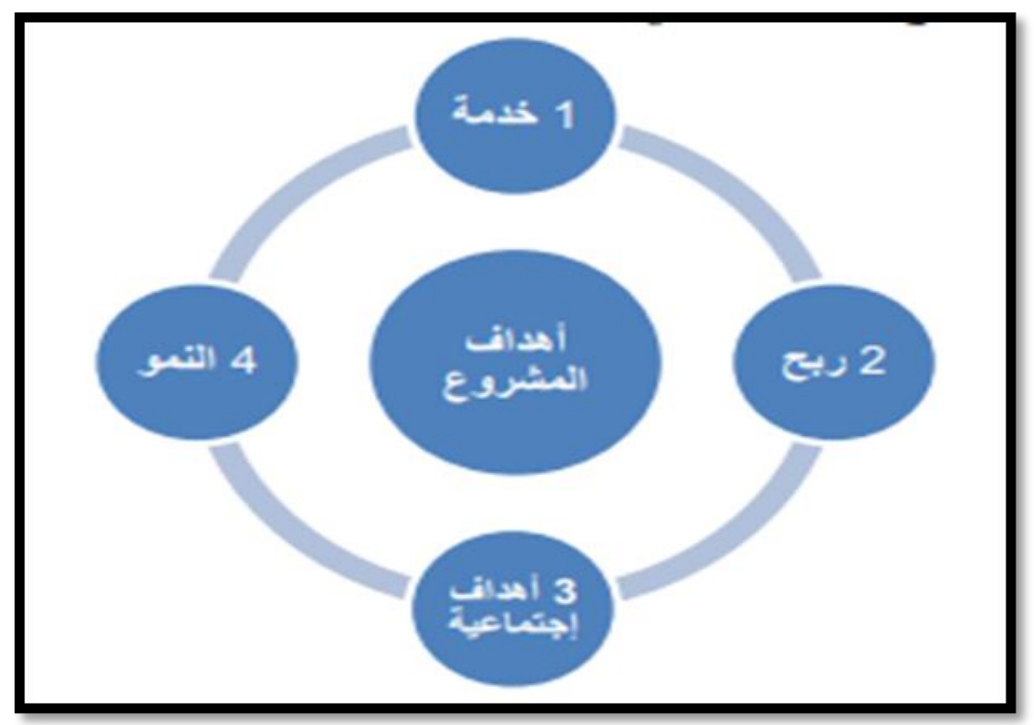

الشكل التوضيحي 3: الأهداف العامة للمشروع (أبو غانم، 2002) 


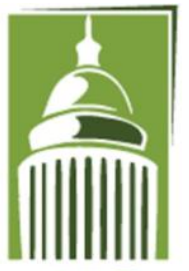

ARF
Global Proceedings Repository American Research Foundation

ISSN 2476-017X

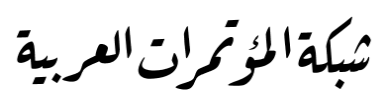

http://arab.kmshare.net/

Available online at http://proceedings.sriweb.org

وخلال العصور الماضية شهدت إدارة المشاريع نمواً ملحوظاً لاعتبارها أداة ووسيلة أساسية في تحقيق أهداف المنظمة، حيث أشار عنبر (2016) إلى أنَّ إدارة المشاريع تُمثل قوة فعالة يُكن من خلالها تحسين قدرة المنظمة على التخطيط والتنظيم والمراقبة، وأضاف دودين (2012) إلى أنَّ مفهوم إدارة المشاريع يشمل جميع الأدوات والوسائل التي يُمكن من خلالها انجاز المشروع خلال الوقت والتكلفة والجودة المطلوبة.

بشكل عام ، يمكن الحكم على نجاح المشروع من أنه تم الانتهاء منه خلال الوقت والتكلفة والجودة المحددة. ومع ذلك، يُيبن تيرنر (Turner, 2009) الوقت المناسب، ولكن بعد خمس سنوات تم الحكم عليه بالفشل. وأكد تنص تيرنر (Turner, 2009) على أن أصحاب المصلحة المختلفين مثل؛ الجهات الراعية والمستخدمين ومديري المشاريع يهكمون على نجاح المشروع بطرق مختلفة، وبالتالي من المهم تحقيق توازن بين تلك المعايير المختلفة لتلبية احتياجات مختلف أصحاب المصلحة (Turner, 2009). وأضاف كيرزنر (Kerzner, 2009) تسليمها في الوقت المحدد وضمن متطلبات التكلفة في تحقيق الأرباح، ولكن في الوقت نفسه قد لا لا يتم التمكن من تحديد ما إذا كان المشروع نفسه قد تمت إدارته بشكل صحيح (Kerzner, 2009).

بالإضافة إلى التعريفات التقليدية لنجاح المشروع يُشير تيرنر (Turner, 2009) إلى وجود بجموعة من المعايير للحكم على نجاح المشروع، والتي تتضمن:

$$
\text { 3 } 1 . \text { 3. يزيد المشروع من قيمة المساهم في المؤسسة الأم. }
$$




\section{Global Proceedings Repository American Research Foundation}

ISSN 2476-017X

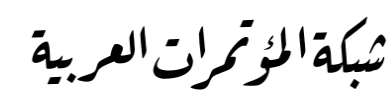

http://arab.kmshare.net/

AR F

Available online at http://proceedings.sriweb.org

$$
\begin{aligned}
& \text { 5. ينتج الأصل الجديد منتجًا أو يقدم خدمة يرغب المستهلكون في شرائها. } \\
& \text { 6. الأصل الجديد سهل التشغيل. } \\
& \text { 7. الانتهاء من المشروع في الوقت المحدد، وضمن الميزانية، ومع الجودة المطلوبة. } \\
& \text { 8. تمتُع فريق المشروع بتجربة مرضية وقد تمكَّن المشروع من تحقيق احتياجاقم. } \\
& \text { 9. تحقيق المقاولون للربح. } \\
& \text { الججالات المعرفية لإدارة المشاريع }
\end{aligned}
$$

لقد حلَّد المعهد الأمريكي لإدارة المشاريع (PMBOK) في الإصدار السادس العمليات الرئيسية الخمس في إدارة المشروع والتي تشمل : بداية الانطلاق Initiating، التخطيط Planning ، التنفيذ Execution ، والمراقبة Control

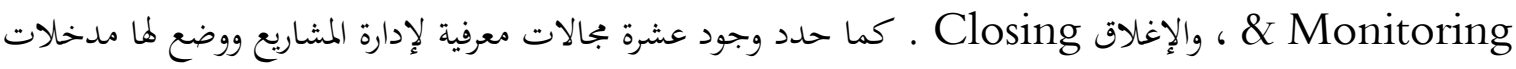
وأدوات ومخرجات، وسنتناول في الجزء التالي تسليط الضوء باختصار على هذه المجالات المعرفية والتي تشمل ما يلي:

1. إدارة تكامل المشروع Integration Management: والتي تتضمن عمليات المشروع منذ البداية وحتى النهاية، وتُعَّف بأها عملية تنسيق جميع الجوانب المرتبطة بالمشروع ومتابعتها وتقييمها بشكل مستمر بصورة يُمكن من خلالما تحقيق رضا العملاء ومصلحة المنظمة (Heldman, 2009). كما يُعرِّها ريتشمان () Richman, 2014) بأغها عملية تتضمن إدارة كافة نشاطات المشروع مع مراعاة دمج جميع الأنشطة مع بعضها وتحقيق تكاملها. ولقد ذكر الميناوي (2014) مجموعة من النشاطات المتواجدة ضمن إدارة تكامل المشروع، والتي تتضمن؛ وضع وثيقة المشروع، وتصميم خطة المشروع، ووضع خطة تنفيذ المشروع، والتحكم في المشروع، بالإضافة إلى اغلاق المشروع. وهذا يعني أنَّ إدارة تكامل المشروع تُعبِّ عن خطة متكاملة وشاملة للعمليات التي يجب الالتزام بها وتطبيقها من أجل ضمان نجاح المشروع، بحيث يكون الأساس الذي ترتكز عليه هو وجود خطة متماسكة وشاملة ومصممة بطريقة مبتكرة تضمن تنفيذها خلال دورة حياة المشروع. 


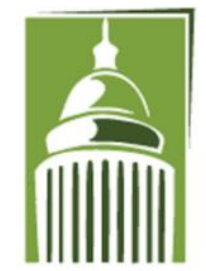

ARF
Global Proceedings Repository

American Research Foundation

ISSN 2476-017X

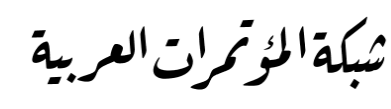

http://arab.kmshare.net/

Available online at http://proceedings.sriweb.org

2. إدارة نطاق المشروع Scope Management: وتعني تحديد كافة العمليات المرتبطة بالمشروع والاستغناء عن الأنشطة الغير مرتبطة بالمشروع (خير الدين، 2012)، وقد تم تعريفها من قبل معهد التحديد القطاعي والمؤسساتي الأوروبي بأها كافة العمليات التي يُمكن من خلالها ضمان نجاح المشروع وتحقيق الجودة المطلوبة له (دليل إدارة نطاق المشروعات، 2008). ولقد أشار وايزوكي (Wysocki, 2014) إلى النشاطات التي يتضمنها إدارة نطاق المشروع، والتي تشمل؛ تحديد الاحتياجات والمتطلبات، وتحديد هيكل العمل، وتعيين النطاق وضبط النطاق. 3. إدارة زمن المشروع Time management: وهي العملية التي تتمثل في تحويل خطة المشروع ضمن اطار زمني Rُمكن من خلاله ضمان انجاز المشروع خلال المدة المحددة (دودين، 2012)، ويُعرّفها ريتشمان ( Richman, 2014) بأها العملية التي يتم من خلالما استغلال الوقت بكفاءة واتمام المشروع خلال المدة المحددة. ويُشير يُمَّمُ (2012) إلى أنَّ إدارة زمن المشروع تُمثل دليلاً للوقت المحدد لتنفيذ أنشطة المشروع في موقع العمل، ويُضيف العلي (2011) إلى أها تُشير تقدير الوقت الذي تتطلبه كل عملية وكل نشاط من الأنشطة المحددة. 4. إدارة تكلفة المشروع Cost Management: وهي التي يُعبَّر عنها بدراسة الجدوى في المشروع (الدراسة الأولية)، بحيث تتضمن القيام بوضع تقديرات لكل نشاط وعملية وتحديد النفقات والايرادات وحساب العائد المتوقع من تنفيذ المشروع (العلي، 2011)، وبيَّن الميناوي (2014) بأنَّ إدارة تكلفة المشروع تضمن اتمام المشروع ضمن الكلفة المحددة دون بحاوزها.

5. إدارة جودة المشروع Quality Management: وهي العملية التي يتم من خلالما ضمان مراقبة الأعمال والتأكد من جودمَا باستخدام وسائل وأساليب متعددة، كما أها تتضمن عملية تقييم المشروع للتأكد من سريانه وفق المطلوب (العلي، 2011)، وتتضمن كذلك اجراء التحسينات المطلوبة بناءً على نتائج التقييم بما يُسهم في تحسين جودة المشروع وجودة المخرجات (دهليز وعنبر، 2017). 


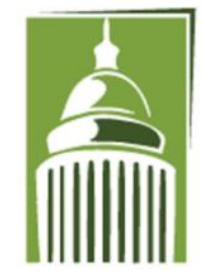

ARF

\section{Global Proceedings Repository}

American Research Foundation

ISSN 2476-017X

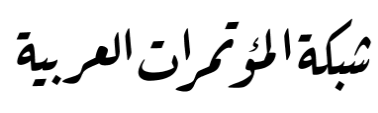

http://arab.kmshare.net/

Available online at http://proceedings.sriweb.org

6. إدارة موارد المشروع Resources Management: وهي العملية التي يتم من خلالاما تزويد موقع العمل بالموارد والمستلزمات اللازمة لتنفيذ المشروع في الوقت المحدد وضمن التكلفة المحدة، حيث بيَّن دهليز وعنبر (2017) أنه من مسؤولية مدير المشروع الاتفاق والتعاون مع أفراد مُككن الاعتماد عليهم في ايصال الموارد المطلوبة في الوقت المحدد. وتتضمن هذه العملية تحديد نوع المورد والكمية المطلوبة والوقت الذي يجب فيه ايصال الموارد إلى موقع العمل دون تأخر

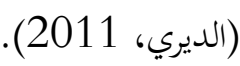

7. إدارة اتصالات المشروع Communication Management: وهي العملية التي تضمن ايصال المعلومات المطلوبة لكافة أفراد المشروع في الوقت المناسب (الميناوي، 2014)، كما أها تتضمن التغلب على المخاطر التي يُكن مواجهتها من خلال ايصال المعلمات في الوقت المناسب لصناع القرار.

8. إدارة مخاطر المشروع Risk Management: وهي العملية التي تتضمن تشخيص المخاطر وتحليلها وتقييمها والاستجابة لها وتسين طرق التعامل معها (المقداد، 2011)، حيث بيَّن العامري (2007) أن عملية إدارة غخاطر المشروع تُمكِّن المدراء من مواجهة حالات عدم اليقين التي تظهر ضمن البيئة المتغيرة، والتي يجب السيطرة عليها لضمان سير تنفيذ المشروع وفق المطلوب.

9. إدارة المشتريات في المشروع Procurement Management: وتتضمن الحصول على السلع والموارد من المصادر المثفق معها ضمن الوقت المناسب والتكلفة المناسبة، كما تستلزم ضبط عملية الشراء والتعامل مع الموردين المناسبين ومراجعة الطلبات بشكل مستمر ودوري (الميناوي، 2014)، وقد جادل عبد العزيز (2008) بأنَّ عملية إدارة المشتريات تتطلب بمموعة من الاجراءات، والتي تشمل: أ. اقامة علاقة طيبة مع الموردين. ب. الشراء بكميات كبيرة من أجل مواجهة حالات الطوارئ. ت. الارتباط مع أكثر من مصدر توريد واحد. 


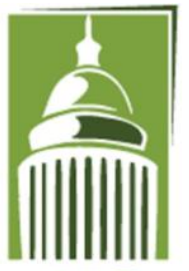

ARF
Global Proceedings Repository American Research Foundation

ISSN 2476-017X

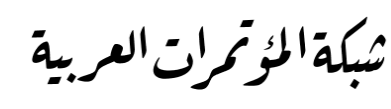

http://arab.kmshare.net/

Available online at http://proceedings.sriweb.org

$$
\begin{aligned}
& \text { ث. وجود فريق قادر على التفاوض مع الموردين من أجل الحصول على أقل سعر. } \\
& \text { ج. تأمين الحد الأدنى من احتياجات ومستلزمات المشروع. }
\end{aligned}
$$

10.إدارة أصحاب المصلحة Stakeholders Management: مفهوم اصحاب المصلحة يشير إلى الأطراف

المعنية بالمشروع سواءا كانوا أفراد أو منظمات أو مجموعات شبكات الذين يؤثرون ويتأثرون بلمشروع ونتائجه، وإدارقم هي عملية تحديد المعنيين بالمشروع بانتظام وتحليل وتوثيق المعلومات ذات الصلة المتعلقة باهتماماتم ومشاركتهم وارتباطاقم وفاعليتهم و تأثيرهم ومشاركتهم في تنفيذ المشروع وفاعليتهم المتملة على نجاح المشروع. و المننعة الأساسية لهذه العملية في أهما تسمح لفريق المشروع بتحديد التركيز المناسب من مشاركة كل المعنيين بالمشروع ، وتؤدى بشكل دوري على مدار المشروع حسب الحاجة. وتتضمن قدرة مدير المشروع على التعامل مع يختلف الأفراد المتواجدين كأطراف معنية في المشروع، والذين يشملون كل من العاملين، والمدراء، والمالكين، والعملاء، والموردين، والمنافسين، والمجتمع الخيط والجهات

$$
\text { الحكومية والدائنين.....أخ. }
$$

لذلك أعتقد كباحث بأن إدارة هذا الجانب المعريف في إدارة المشاريع ذو أهمية بالغة وذلك لكونه بمثل العنصر البشري الحاضن لرأس المال الفكري وللمعرفة الضمنية وكذلك لتأثيره الفاعل في تنفيذ العمليات الأساسية لإدارة المشروع ولإدارة الجوانب المعرفية العشرة للمشروع ويكنن القول بأنه لاوجود لإدارة المشروع أو المعرفة بدون وجود اصحاب المصلحة.

\section{إدارة المعرفة تضمن قيمة المملومات في المشروع:}

أشار ريتشي وجورقينسن (Ritchie, P. \& Jorgensen, K. 2007) في دراسة بخثية لمما إلى أهمية البيانات والمعلومات في ادارة المشاريع، وركزا على الحد الأدن من سياسة معلومات المشروع القياسية والمعيارية. وتحدد هذه السياسة الحد الأدن من المعلومات التي سيتم الاحتفاظ بما حول مشروع ما وبالتالي مككن تطبيقها لاحقا على جميع المشروعات، والغرض من هذه السياسة هو ضمان الحصول على الحد الأدنى من معلومات المشروع وتزينها من أجل: 


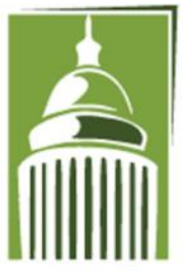

ARF

\section{Global Proceedings Repository \\ American Research Foundation}

ISSN 2476-017X

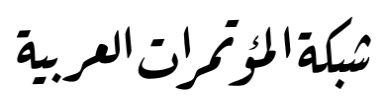

http://arab.kmshare.net/

Available online at http://proceedings.sriweb.org

$$
\text { تقديم معلومات المشروع لمساعدة ممارسي إدارة المشروع في إدارة المشروع. }
$$

توفير معلومات المشروع لصناع القرار في الإدارة الاستشارية وغيرهم من أصحاب المصلحة والأطراف المعنية في المشروع.

$$
\text { تقديم معلومات المشروع لتلبية المتطلبات القانونية. }
$$

قديم معلومات المشروع لتلبية متطلبات إعداد لوحة وخارطة المعلومات.

$$
\text { تقديم معلومات المشروع لدعم إدارة المعرفة. }
$$

ولدعم إدارة المعرفة ، تحدد سياسة الحد الأدنى لمعلومات المشروع متطلباته بشيء من التفصيل .وهذا الوضوح يعيد في الواقع وضع التوقعات بشأن المعلومات التي يجب على مديري المشاريع إنتاجها وتزينها وحفظها في مشاريعهم .بالإضافة إلى ذلك ، تميز هذه السياسات أيضًا بين ثلاثة أنواع من البيانات أو المعلومات التي يجب على مديري المشاريع إنتاجها وتخزينها وحفظها. هذه الأنواع هي سمات المشروع المخزنة في قواعد البيانات (المعلومات الهيكلية) Structured information ، ووثائق المشروع التي يمكن الوصول إليها كمرجع (المعلومات شبه المنظمة) Semi-Structured information ، والمعلومات المستندة إلى الخبرة أو Explicit Tacit information الممارسة (المعلومات الضمنية) حيث يشير هذا التقسيم إلى أهمية المعرفة الصريحة الظاهرة المتوفرة في المعلومات الموثقة والمدونة في وثائق وسجلات وقواعد بيانات عمليات المشروع الرئيسية وجوانبه المعرفية العشرة، هذا بالإضافة إلى المعرفة الضمنية Tacit Knowledge الكامنة في عقول الحبراء والمستشارين والمدراء واصحاب المصلحة والأطراف المعنية ذات المهارات والخبرات والمعرفة المتخصصة والتي تحتاج إلى مزيد من الاهتمام والتوثيق والتحويل إلى معارف صريحة يمكن الاستفادة منها في إدارة المشاريع المستقبلية كما هو موضح في الشكل التالي الذي قدمه ريتشي وجورقينسن .(Ritchie, P. \& Jorgensen, K. 2007) 


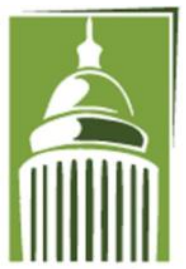

ARF
Global Proceedings Repository

American Research Foundation

ISSN 2476-017X

Available online at http://proceedings.sriweb.org

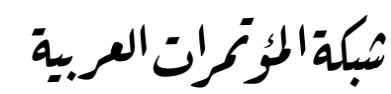

http://arab.kmshare.net/

\section{Structured Information}

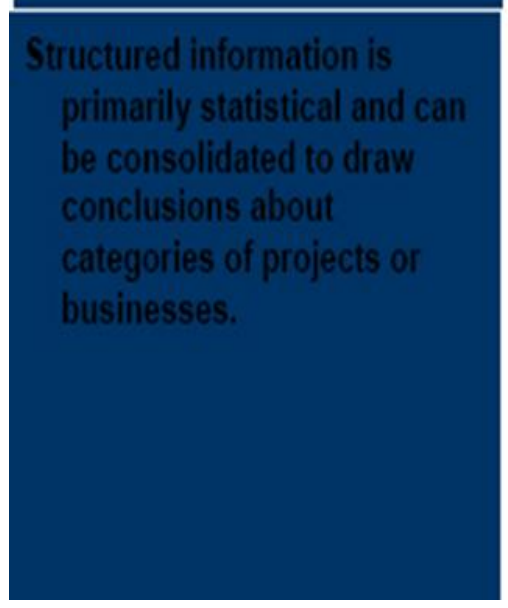

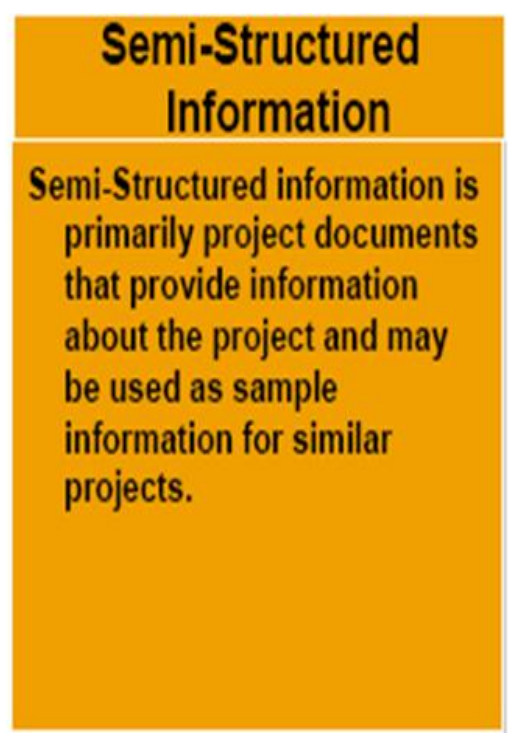

\section{Tacit Information}

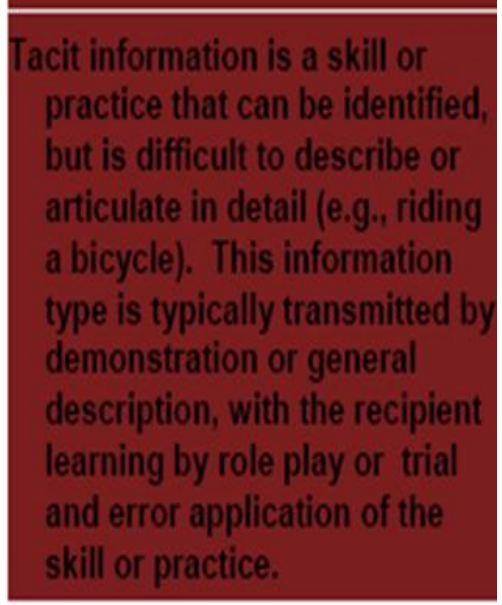

الشكل التوضيحي = 4: تعريف نوع معلومات إطار العمل الخاص بـ KM Framework

وتبدأ العملية كما أوضح ريتشي وجورقينسن بضرورة تخزين مواد المعرفة "المعلومات الخام" من مختلف الأنواع التي تمر من خلال تنفيذ

عمليات المشروع وجوانبه المعرفية عبر مجموعة هيكلية شفافة من العمليات تشمل التخزين ثم التحضير والامتلاك والتعديل ثم المشاركة لإنشاء معلومات ذات قيمة مضافة وميزة تنافسية عالية كما يعرض الشكل التوضيحي أدناه:

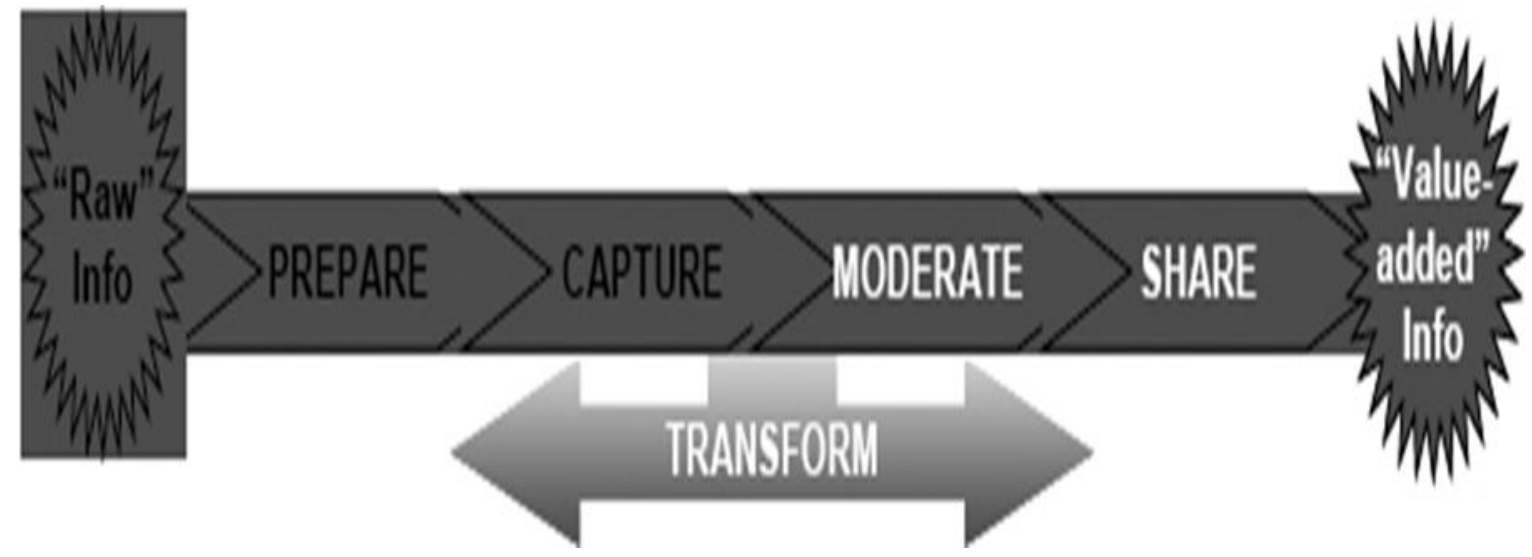

الشكل التوضيحي- 5 : إطار عمل KM لتحويل استخدام المعلومات الخام المشتركة في المشاريع. 


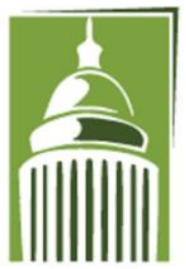

ARF
Global Proceedings Repository

American Research Foundation

ISSN 2476-017X

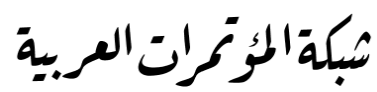

http://arab.kmshare.net/

Available online at http://proceedings.sriweb.org

وبعد إجراء العمليات السابقة على مواد المعرفة المتمثلة في المعلومات والبيانات المهمة للمشروع ومن ثم انتاج القيمة المضافة لها لتمنح المشروح ميزة تنافسية تؤهله للنجاح، قام كلا من ريتشي وجورقينسن بتأكيد ضرورة إعادة إرسال ومشاركة المعرفة لنقل الممارسات المحدة الموصى بها، وأفضل الأمثلة والدروس المستفادة ـ ويمكن مشاركة وتبادل المعرفة في إدارة المشروع عبر قنوات الاتصال الأساسية لكل نوع من أنواع مواد المعرفة في المعلومات المذكورة سابقا، حيث يمثل لون قناة الاتصال نوع المعرفة الأساسي الذي يتصل به على Tacit Knowledge تمثل اللون الأمر = وهذا يمثل المعرفة الضمنية Message Boards مبثال : لوحات الرسائل هذه ليست علاقات حصرية غير قابلة للتعديل بل تسمح لوحات الرسائل للمساهمين بإرفاق المستندات وهي معلومات شبه منظمة. (Ritchie, P. \& Jorgensen, K. 2007).

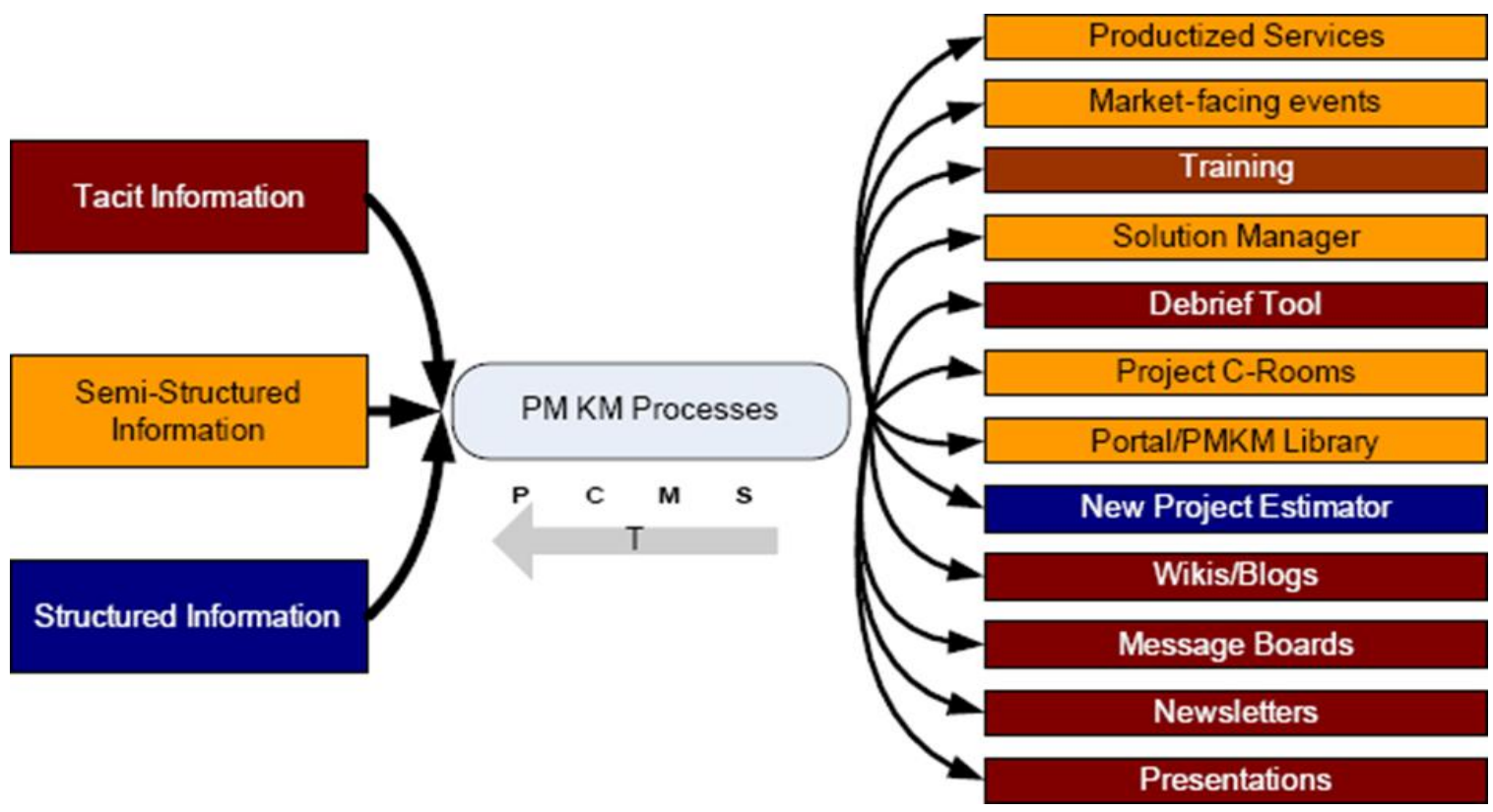

الثكل التوضيحي-6: قنوات الاتصال للحصول على معلومات القيمة المضافة

أهمية اندماج عمليات ادارة المعرفة مع عمليات ادارة المشاريع : 


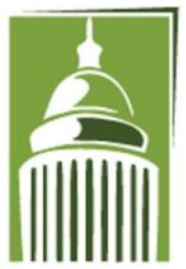

ARF
Global Proceedings Repository

American Research Foundation

ISSN 2476-017X

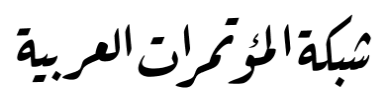

http://arab.kmshare.net/

Available online at http://proceedings.sriweb.org

تعد مكونات PM و KM متشاهة جدا. حيث تشمل مكونات PM : النظام والأشخاص والأدوات وتشمل مكونات KM الأشخاص والتكنولوجيا والعوامل التنظيمية، وحيث أن المكونات متشاهة، فإنه يمكن القول بأن العنصر البشري يشكل العنصر القيادي والمحور الرئيسي الذي تعتمد عليه إدارة المشاريع وكذا الحال بالنسبة لإدارة المعرفة، ويشكل العنصر البشري المصدر المولد والمنتج للمعرفة الضمنية والخبرات المتخصصة والمهارات القيادية الفاعلة لإدارة المشاريع بشكل يضمن نجاح المشروع وتكامل العمليات فيه. كما أن للمكونات من كلا المجالين يسمح بوضعها فوق بعضها البعض، وقد أوضحها ريتشي وجورقينسن بحيث يمكن دبجها والعمل مع بعضها البعض كما هو مبين في الشكل (Ritchie, P. \& Jorgensen, K. 2007)

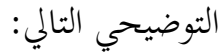

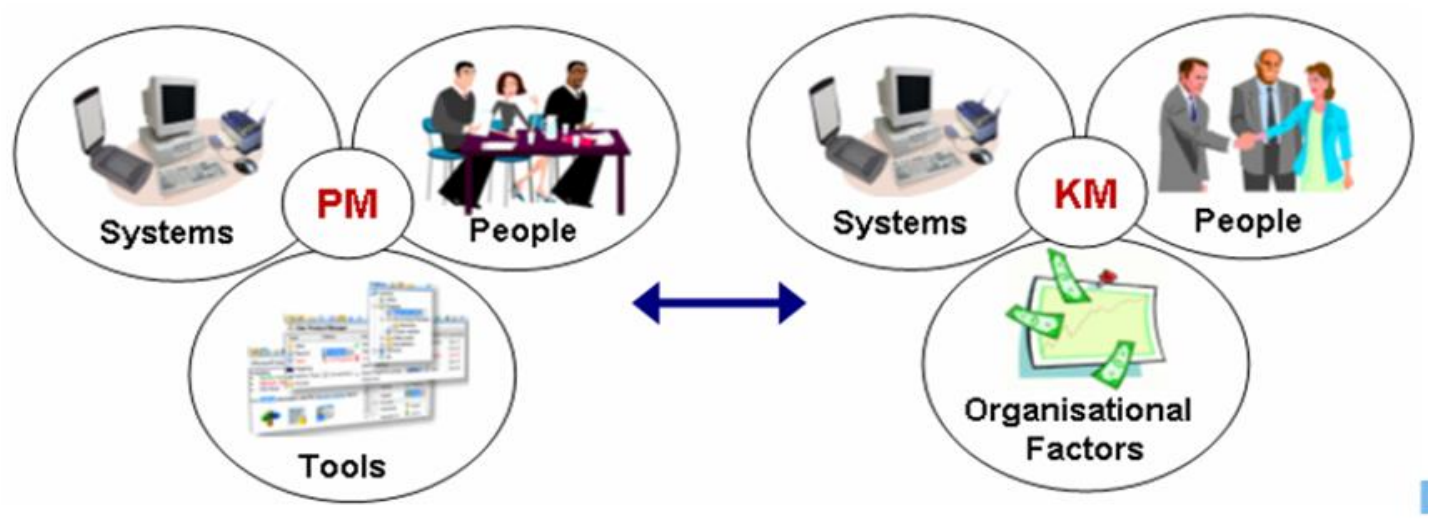

Figure 3. Knowledge Management and Project Management components

$$
\text { الشكل التوضيحي-7: الكامل مكونات KP و KM }
$$

لقد تضمَّن الأدب النظري العديد من النماذج والأطر التي حاولت تفسير العلاقة بين عمليات إدارة المعرفة وأهميتها في المجالات المعرفية لإدارة المشاريع، حيث بيَّن كوب وآخرون (Cope et al., 2006) أن إدارة المعرفة هي ممارسة منطقية لتحسين إدارة 


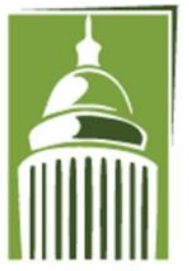

ARF
Global Proceedings Repository American Research Foundation

ISSN 2476-017X

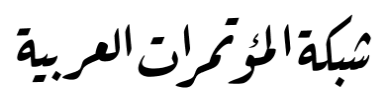

http://arab.kmshare.net/

Available online at http://proceedings.sriweb.org

المشروع، وأََّد على أنه إذا أمكن الحصول على المعرفة (النماذج الضمنية والصريحة) ومشاركتها في بجتمع إدارة المشروع فإن المنظمات ستستفيد كثيرًا.

وبالعودة إلى ما تم الإشارة له سابقا في نموذج دورة إدارة المعرفة (SECI) لنوناكا وتاكيوتشي، فقد أشار بوليانينوفا بأن إدارة المعرفة KM تسمح بالحصول على المعرفة الضمنية النابحة عن الخبرة الجماعية في (Polyaninova, 2011) المشروع ونشرها في أي مكان في العمل، لذلك قام بتوضيح بعضا من العمليات والأدوات اللازمة في إدارة الجوانب المعرفية المختلفة للمشروع وإدخال تكاملها في التفاعل الضمني والصريح الموضح في شكل نموذج لولب المعرفة (KSM) أدناه، وبين أنه يتم اكتساب المعرفة الضمنية من خلال التعلم ، والخبرة ، والحس السليم ، والقيم والمعتقدات. تعتبر المعرفة الضمنية أكثر قيمة، على الرغم من أنه من الصعب التقاطها في شكل مسجل أو موثق أو مقنن ويتم الكشف عن المعرفة الضمنية من خلال تطبيقاتما المختلفة. نقل المعرفة الضمنية بين الناس أمر صعب وبطيء ومكلف وغير مؤكد بخلاف المعرفة الصريحة فهي قابلة للتدوين والتوثيق ويمكن نقلها بلغة رسمية. على سبيل المثال: سجلات المشروع والأدلة والوثائق والتقارير ومن السهولة نقلها ومشاركتها وتسهيل التواصل فيما بين أعضاء فريق المشروع لتقييم المعرفة الصريحة وتنظيمها وإتاحتها للأشخاص الذين يمكنهم استخدامها لدعم عمليات المشروع المختلفة. ولذلك (Polyaninova, 2011). 


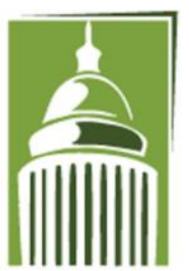

ARF

\section{Global Proceedings Repository \\ American Research Foundation}

ISSN 2476-017X

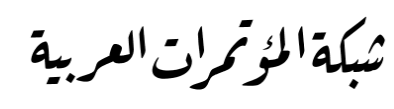

http://arab.kmshare.net/

Available online at http://proceedings.sriweb.org

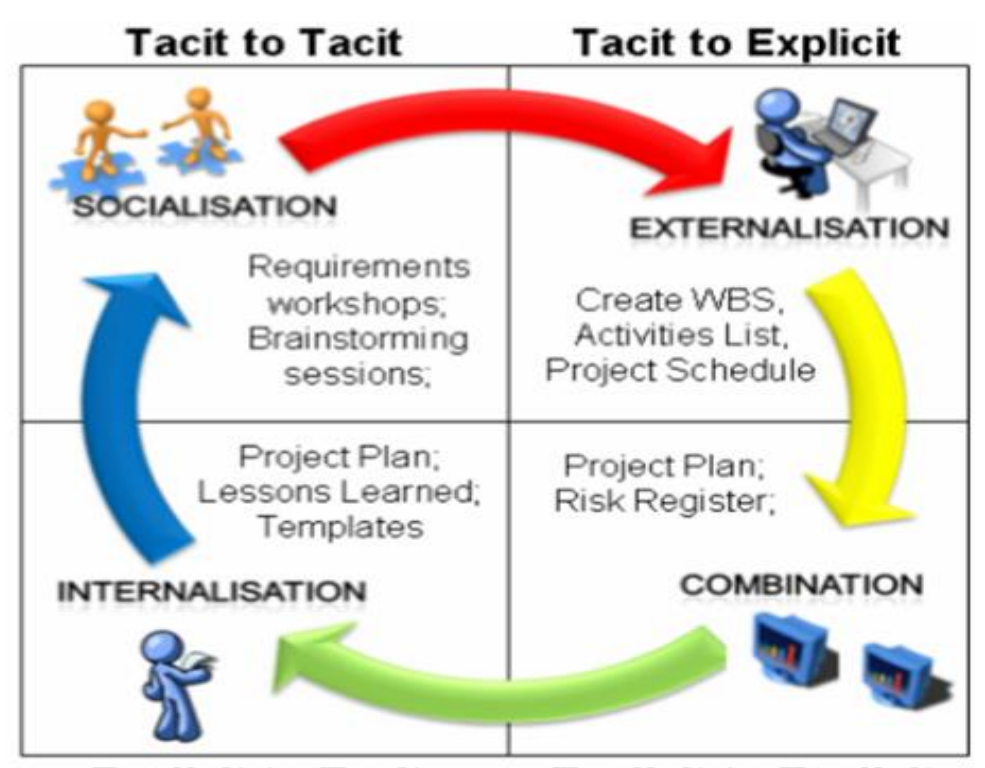

Explicit to Tacit

Explicit to Explicit

Figure 2. Knowledge Spiral model and project activities

الشكل التوضيحي- 8: إدخال التفاعل الضمني والصريح في شكل نموذج لولب المعرفة مع إدارة المشاريع (KSM)

يعمل KSM جنبًا إلى جنب مع مراحل المشروع كما هو موضح في الشكل الذي قدمه بوليانينوفا. على سبيل المثال ، تحدث عملية "التنشئة الاجتماعية أو التفاعل الاجتماعي" SSM S Socialization) في المشروع خلال اجتماعات المشروع، ومراجعات ما بعد المشروع ، وجلسات العصف الذهني، وحلقات العمل وتقييمات المشروع. إذا كان هناك خلال عملية "التنشئة الاجتماعية أو التفاعل الاجتماعي" للمشروع مقاومة أو اكتناز أو احتكار للمعرفة ، فإن المعرفة تصبح راكدة. وخلال العملية التالية " التجسيد" KSM J I Externalization) ، يتم تدوين المعرفة الضمنية للمشروع وتحويلها لمعرفة صريحة من خلال إنشاء جدول المشروع أو قائمة الأنشطة أو WBS ويمكن دمج المعرفة الصريحة أثناء عملية "الجمع" لـ KSM ، على سبيل المثال من خلال إنشاء خطة المشروع التي تجمع جميع خطط المثاريع الأخرى ، مثل خطة إدارة المخاطر وقائمة الأنشطة وخطة إدارة 


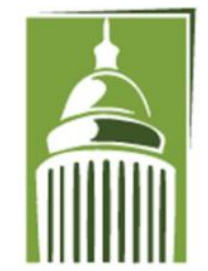

ARF

\section{Global Proceedings Repository \\ American Research Foundation}

ISSN 2476-017X

\section{شبكة المؤمرات العربية}

http://arab.kmshare.net/

Available online at http://proceedings.sriweb.org

النطاق والجدول الزمني للمشروع. يقوم الأفراد بمراجعة المعرفة المقننة وتحويلها من معرفة صريحة إلى ضمنية أثناء عملية " التذويب" KSM J(Internalization) امتلاكها واكتسابها وتكوين معرفة ضمنية جديدة يمكن تطبيقها في تنفيذ المشاريع المستقبلية.

ومع نضوج بيئة المشروع داخل المنظمة، تنضج عملية تكامل إدارة المعرفة وإدارة المشاريع أيضًا. ومع تقدم المشروع ، تنتقل المعرفة من ضمني إلى صريح وتعود إلى المعرفة الضمنية من خلال KSM ، وهذا بالتأكيد يعد أمر حيوي لضمان أن المعرفة يتم تداولها بشكل صحيح من خلال الدورة التي تسمح بتحديد متطلبات المشروع وشرحها وتسليمها مع تسليم المنتج النهائي للعميل أو المستفيد وفقًا للمتطلبات المحددة في بداية المشروع. من الضروري ضمان عدم ضياع المعرفة بين مراحل المشروع وأثناء دمج المراحل. يحدث هذا ، على سبيل المثال ، عندما يتم تعيين فريق عمل وأشخاص مختلفين للعمل في مشروع، أو تحديد أصحاب مصلحة وأطراف معنية مختلفة، قد يشعرون أن ما يملكونه من معارف ضمنية هي معرفتهم الخاصة ولا يميلون لنقلها وتبادلها ومشاركتها مع الآخرين إلا عبر حوافز مادية ومعنوية محددة. أو ربما يكون الجدول الزمني والمواعيد النهائية لتسليم المشروع لا تسمح بوقت كاف لتوثيق المعرفة ونقلها فعليًا. وربما تظهر بعض المتطلبات الإضافية خلال فترة تنفيذ المشروع ، مما يخلق قيودًا على الميزانية والدعم المالي تحد من بذل مزيد من النفقات مما يكون له آثار على إدارة المعرفة داخل المشاريع.

وأشار اسماعيل وآخرون (Ismail et al., 2009) على أنه وعلى الرغم من وجود العديد من الأدب النظري المرتبط بتبادل المعرفة ، إلا أنه لا يُعرف الكثير عن كيفية مشاركة الأفراد للمعرفة، خاصة في بيئة المشروع. ومن هنا اقترح اسماعيل وآخرون إطارًا نظريًا يشير إلى أن توفير الدوافع المناسبة وإزالة العوائق التي تمنع وتحد من امكانية تبادل المعرفة (Ismail et al., 2009) والخبرات من شأنه أن يؤدي إلى مشاركة أكثر كفاءة وفعالية للمعرفة في المشاريع والتي بدورها ستؤدي إلى زيادة الاحتمال نجاح المشروع. كما يُبِين النموذج الخاص إلى وجود علاقات مهمة بين ممارسة المشاركة الفعالة في معرفة المشروع ونجاح المشروع. وبشكل أساسي استند هذا النموذج إلى نموذج تحويل المعرفة وإدارتحا المعروف باسم نموذج دورة إدارة المعرفة (SECI)، والذي يركز على 


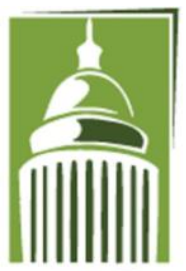

ARF
Global Proceedings Repository

American Research Foundation

ISSN 2476-017X

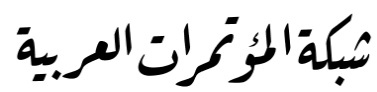

http://arab.kmshare.net/

Available online at http://proceedings.sriweb.org

التنشئة الاجتماعية للمعرفة الضمنية التي تشكل حاليًا فجوة في معظم بيئات المشروع. وبناءً على ذلك استنتج اسماعيل وآخرون (2009) أن ضمان الوقت والكيفية التي يتم من خلالها تبادل المعرفة الضمنية والصريحة يُعتبر أمر ضروري لتعزيز نجاح المشروع. الشكل التالي يوضح الاطار النظري المقترح في مساهمة مشاركة المعرفة في نجاح المشروع.

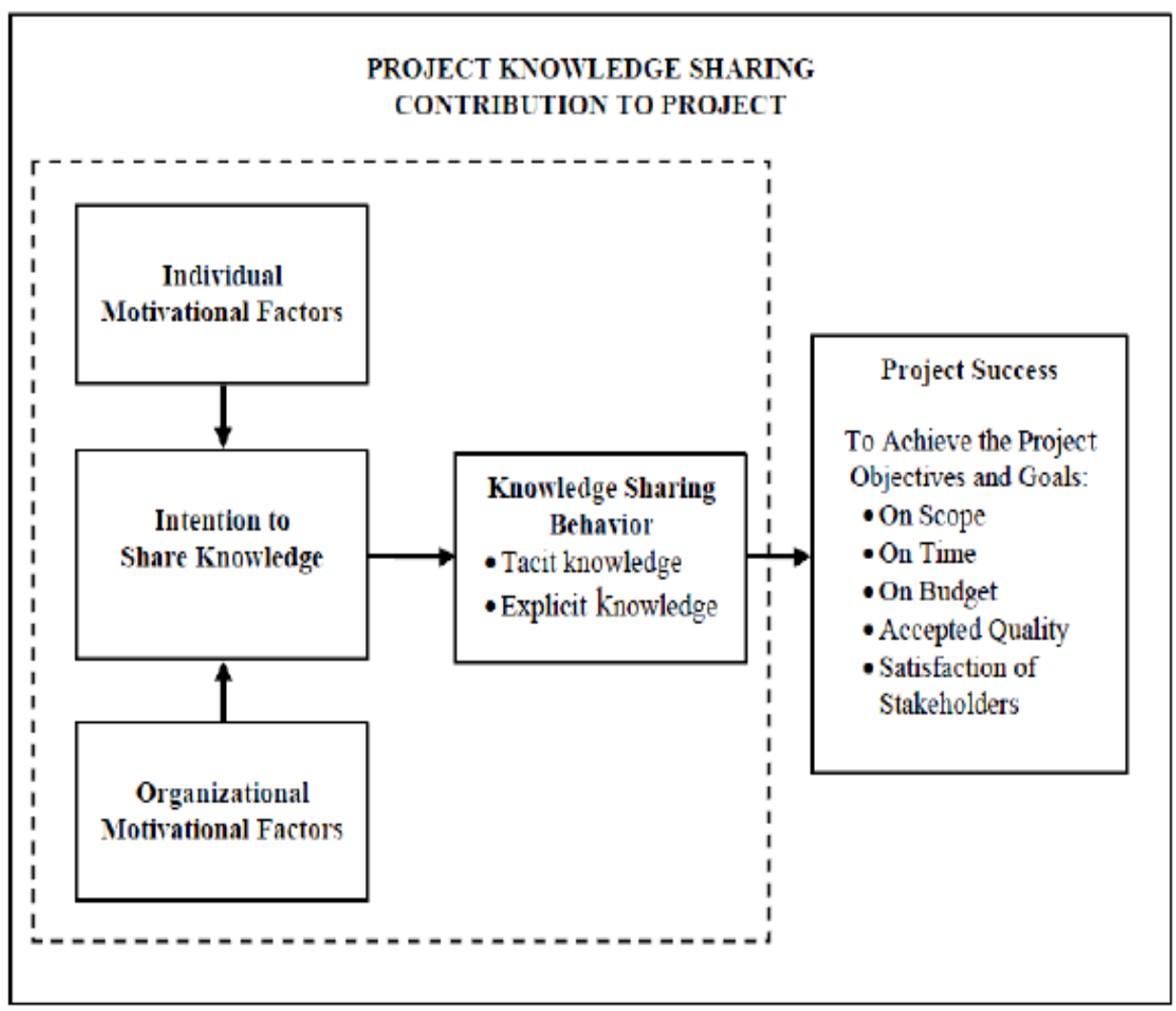

الشكل التوضيحي- 9: يوضح الاطار النظري المقترح في مساهمة مشاركة المعرفة في نجاح المشروع (Ismail et al., 2009)

قدَّم أوين (Owen, 2008) أطاراً آخراً لتوضيح أهمية توظيف إدارة المعرفة في تحقيق تكامل ونجاح المشاريع، حيث أشار في نموذجه إلى أنَّ المعرفة التي تم إنشاؤها ونقلها والتقاطها وإعادة استخدامها داخل المشروع سوف تؤدي إلى تحسين إدارة المشروع. كما قدم أوين (Owen, 2008)من خلال هذا النموذج هيكلاً لربط إدارة المشروع بإدارة المعرفة وتحقيق 


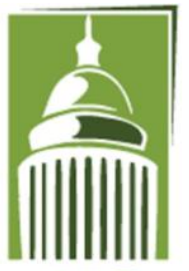

ARF
Global Proceedings Repository American Research Foundation

ISSN 2476-017X

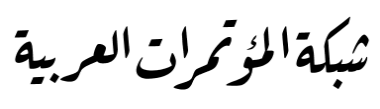

http://arab.kmshare.net/

Available online at http://proceedings.sriweb.org

الاستغلال المتبادل لكليهما (انظر الرسم التوضيحي في الأسفل). وبناءً على ذلك يمكن تعريف المشروع باعتباره مهمة حيث يتم إنشاء المعرفة نتيجة للأنشطة التي تقوم بها فريق المشروع، ويقوم أعضاء فريق المشروع بإنشاء ونقل وإعادة استخدام المعرفة التي تم إنشاؤها من المهام التي يدعمها نظام إدارة المعرفة. كما يشير هذا الإطار إلى أن أعضاء فريق المشروع سوف يكونوا قادرين على تصور المهمة، وإعادة استخدام وتطبيق المعارف والخبرات السابقة التي يدعمها نظام إدارة المعرفة.

ويوضِّح إطار عمل أوين (Owen, 2008) ،كما هو موضح في الشكل التوضيحي أدناه، الطريقة التي يتم من خلالها تطوير المعرفة على مستوى المهمة والتي يتم تضمينها في منهجية المشروع وفي بيئة المشروع وفي النهاية تحسِّن من قدرة المنظمة، حيث أهما تشير إلى أن المعرفة جزء لا يتجزأ من دورة حياة المشروع على كل من المستوى التكتيكي والصريح، بحيث يتم التقاط المعرفة الضمنية وإعادة استخدامها على مستوى المشروع في شكل معرفة شخصية يساهم بها أعضاء فريق المشروع، وبعدها يتم نقل المعرفة الضمنية وإعادة استخدامها من خلال التوجيه من أعضاء المشروع مع المزيد من الخبرة، كما يتم إعادة استخدام المعرفة الصريحة من حيث وثائق المشروع التي تم التقاطها خلال دورة حياة المشروع. وبالتالي استنتج أوين (Owen, 2008) أنه من أجل تقديم مؤسسة ناجحة للمشاريع؛ لا بدَّ من تحقيق التعلم المستمر لتحسين قدرتا، بحيث يمكن اشتقاق التعلم المستمر من حيث وضع مبادئ توجيهية لإنشاء وتبادل وإعادة استخدام المعرفة في بيئة إدارة المشروع، وبالتالي دمج ممارسات إدارة المعرفة مع إدارة المشروع وتحقيق التكامل بينهما. 


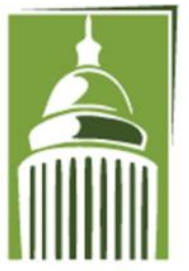

ARF
Global Proceedings Repository

American Research Foundation

ISSN 2476-017X

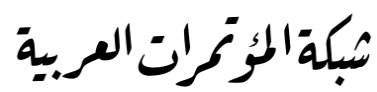

http://arab.kmshare.net/

Available online at http://proceedings.sriweb.org

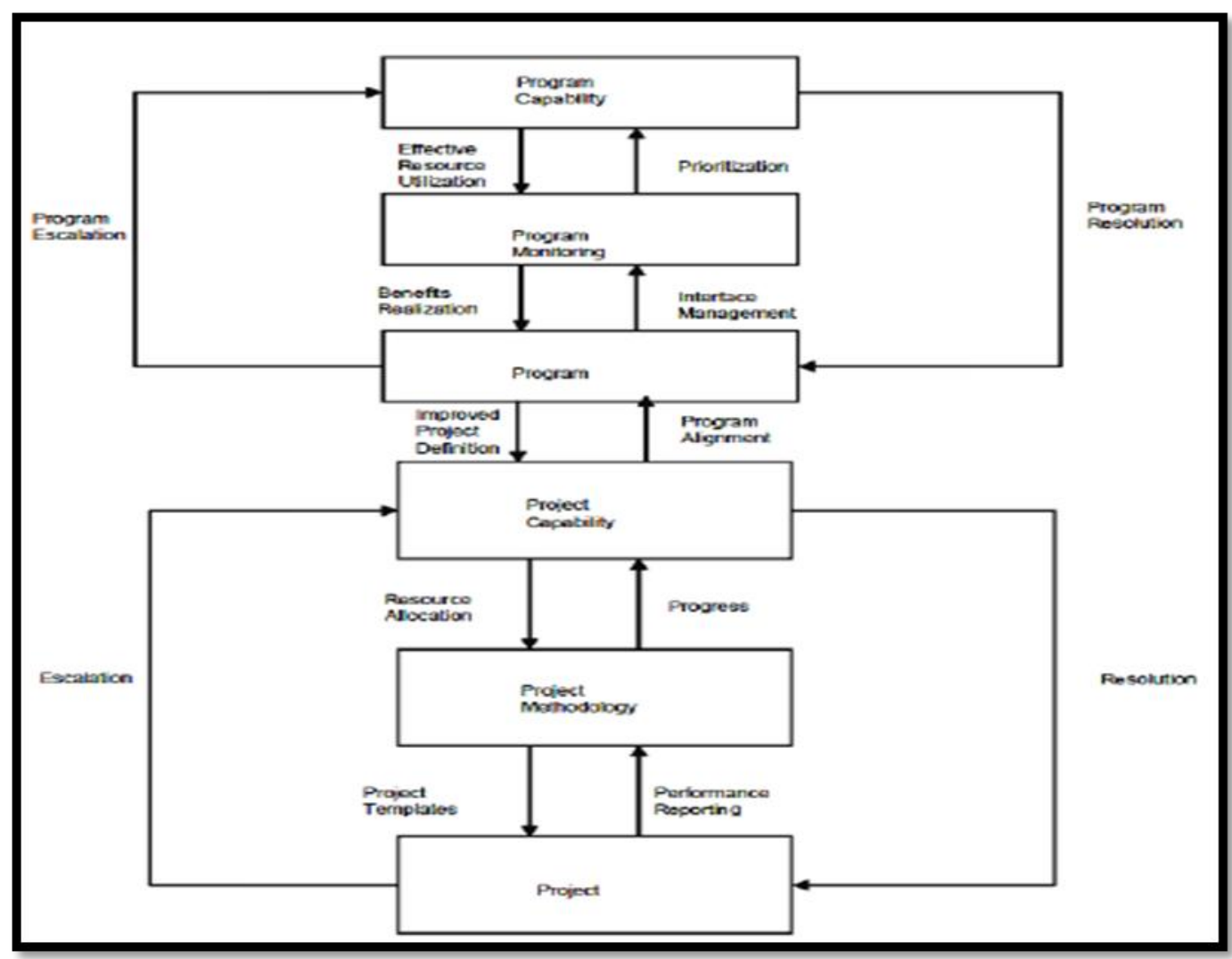

الشكل التوضيحي- 10: اطار توظيف إدارة المعوفة في تحقيق تكامل ونجاح المشاريع (Owen, 2008)

أما جودي وبيكيرا فرنانديز (Gudi and Becerra-Fernandez, 2006) فقد ابتها إلى محاولة دراسة دور إدارة المعرفة في إدارة مشاريع منظمات النظم المعقدة، حيث كان الدافع وراء أبحاثهم هو تقليل المخاطر ومنع الفشل أثناء تطوير أنظمة معقدة والتركيز على الجوانب الديناميكية لإدارة المشاريع باستخدام إدارة المعرفة. يُجادل جودي وبيكيرا فرنانديز بأنه إذا تمكن أفراد المشروع من فهم طبيعة النظم المففوفة بالمخاطر (Gudi and Becerra-Fernandez, 2006) بشكل أفضل، فقد يتمكنوا بالضرورة من الحد من مخاطر الفشل أو إزالتها وزيادة فرصة نجاح المشروع. ويُشير جودي وبيكيرا فرنانديز (Gudi and Becerra-Fernandez, 2006) إلى أنه عندما يزداد تعقيد المشروع يصبح من المهم إيجاد 


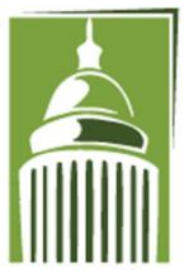

ARF
Global Proceedings Repository American Research Foundation

ISSN 2476-017X

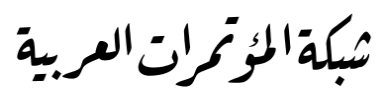

http://arab.kmshare.net/

Available online at http://proceedings.sriweb.org

الوسائل لإدارة الترابط بين المشروعات الفرعية والأنشطة والأحداث ذات الصلة في بيئات المشروع للحد من مخاطر الفشل

$$
\text { وزيادة فرصة نجاح المشروع. }
$$

وقام جودي وبيكيرا فرنانديز (Gudi and Becerra-Fernandez, 2006) بتحديد بعض آليات إدارة المعرفة والعمليات والتقنيات التي قد تكون مناسبة لمتطلبات إدارة المشروع، حيث تم تلخيص النموذج المفاهيمي لدور إدارة المعرفة في إدارة المشروع في الشكل التوضيحي الموضح أدناه. واستنتجوا أن هناك العديد من العوامل التي تئثر على مخاطر المشروع في منظمات المشاريع المعقدة، والتي تشمل العوامل الخارجية مثل الآثار السياسية والاقتصادية، والعوامل الداخلية، ومستوى الابتكار في بيئة المشروع.

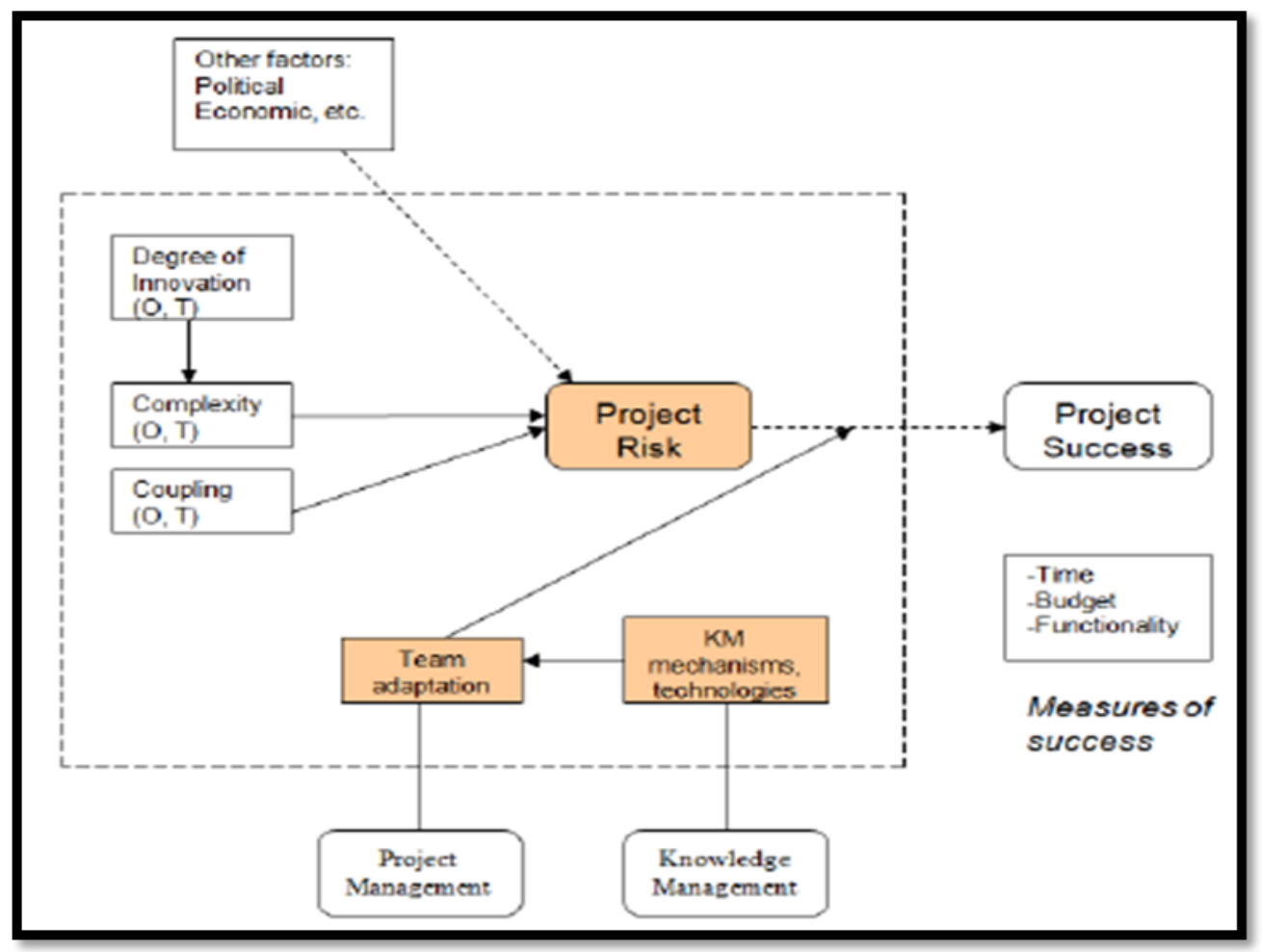

الشكل التوضيحي- 11: النموذج المفاهيمي لدور إدارة المعرفة في إدارة المشروع (Gudi and Becerra-Fernandez, 2006) 


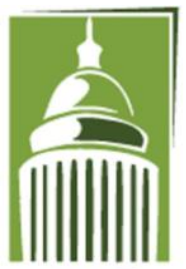

ARF
Global Proceedings Repository

American Research Foundation

ISSN 2476-017X

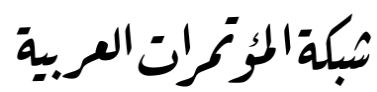

http://arab.kmshare.net/

Available online at http://proceedings.sriweb.org

إضافةً إلى ذلك؛ تمكَّن يونغ وليم (Yeong and Lim, 2010) من تصميم نموذج يوضح أهمية الاندماج بين إدارة المعرفة وإدارة المشاريع، حيث اعتمد الباحثان بشكل أساسي على دراسة نماذج الدراسات السابقة واستحداث نموذج جديد. وبشكل أساسي اعتمد النموذج على ضرورة الزام مدراء المشاريع المشروع بإبداء ملاحظاقم باستمرار ومواءمة المعرفة الموجودة من المخزون والمعرفة التي تم إنشاؤها حديثًا من المشروعات لتعزيز نجاح المشروع، بحيث يمكن القيام بذلك عن طريق بناء العملية لدورة حياة المشروع وعقد مناقشات منتظمة لتبادل المعرفة مع جميع أعضاء المشروع وأصحاب المصلحة كما هو موضح في الشكل أدناه، ويعتمد النموذج على مقترحين أساسيين، الأول يرتكز على اعتبار الثقافة، والعملية، والتكنولوجيا عوامل شائعة تؤثر على إدارة المعرفة وإدارة المشاريع. أما الثاني فيرتكز على أنَّ التغذية الراجعة المستمرة، المواهمة بين إدارة المعرفة وإدارة المشاريع تعمل على تعزيز نجاح المشروع.

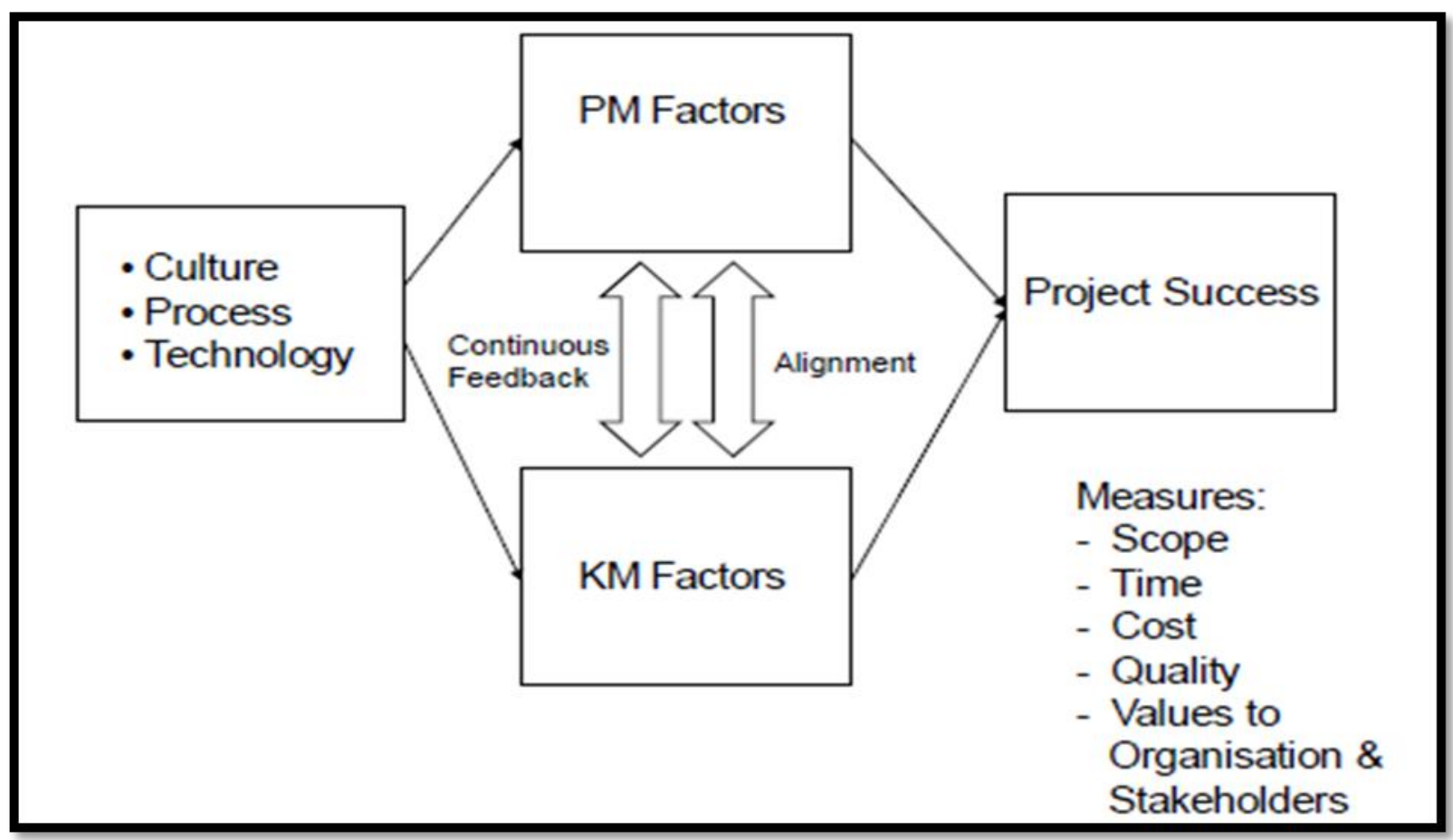

الشكل التوضيحي-12 : أهمية الاندماج بين إدارة المعرفة وإدارة المشاريع (Yeong and Lim, 2010) 


\section{Global Proceedings Repository American Research Foundation}

ISSN 2476-017X

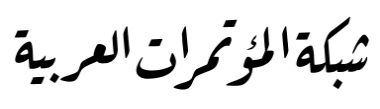

http://arab.kmshare.net/

Available online at http://proceedings.sriweb.org

ومن أجل تعزيز قدرة المنظمات على تحقيق الفعالية المطلوبة من دمج إدارة المعرفة مع المجالات المعرفية لإدارة المشاريع؛ اقترح ليفن تسعة مبادئ توجيهية للمنظمة لتحقيق التنفيذ الناجح لدمج إدارة المعرفة مع إدارة المشروع على النحو التالي: (Levin, 2010)

$$
\begin{aligned}
& \text { 1. ت تحديد إدارة المعرفة بحيث يمكن للجميع في المنظمة فهمها } \\
& \text { 2. جعل إدارة المعرفة حزمة عمل في هيكل توزيع العمل لكل مشروع }
\end{aligned}
$$

3. تأسيس نقطة اتصال لإدارة المعرفة في كل برنامج ومشروع يعمل مع مكتب إدارة مشروع المؤسسة 4. استخدم مصفوفة مسؤولية المساءلة (RAM) لتحديد الأدوار والمسؤوليات والمساءلة لإدارة المعرفة 5. ايصال أهمية إدارة المعرفة لجميع أصحاب المصلحة في جميع أنحاء المنظمة 6. تقديم التوجيه والتدريب المناسب في إدارة المعرفة لجميع أصحاب المصلحة 7. إنشاء نظام عملي لإدارة المعرفة وتقدير المكافآت 8. ت تتبع فائدة إدارة المعرفة باستخدام المقاييس 9. - ينبغي أن تركز المنظمة على التحسين المستمر

بالتالي تؤكد هذه النماذج على أهمية توظيف عمليات إدارة المعرفة في المجالات المعرفية العشرة لإدارة المشاريع وفي قدرتا على تعزيز فرص نجاح المشروع من خلال اتمام المشروع ضمن التكلفة المناسبة والوقت المناسب وضمن الجودة المطلوبة ومن خلال تعزيز التواصل بين الأفراد ونشر المعرفة بينهم. وفي دراسته؛ أكَّد الزيّّات وآخرون (Al-Zayyat et al., 2010) على أنَّ توفر المعرفة الصحيحة للشخص المناسب وفي الوقت المناسب يمنح المدراء سيطرة أكبر على المشروع من خلال دورة حياة المشروع عن طريق الحد من حلال عدم اليقين. بناءً على ذلك تم التوصل إلى أن عمليات إدارة المعرفة الخاصة بتوليد المعرفة وتقييمها هي الأكثر أهمية من حيث تحسين إدارة المشروعات. كما توصل أوليكب وآخرون (Oluikpe et al., 2009) إلى أنَّ إدارة المعرفة تُعتبر عامل أساسي في تسريع وقت إنجاز المشروع، كما ارتبطت كذلك بالابتكار ونجاح المشروع والكفاءة 


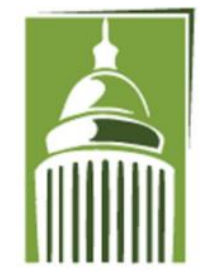

ARF
Global Proceedings Repository American Research Foundation

ISSN 2476-017X

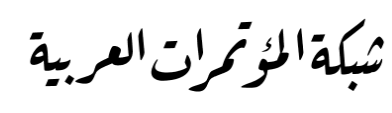

http://arab.kmshare.net/

Available online at http://proceedings.sriweb.org

الخاتمة

ترغب كل مؤسسة في الاستفادة من إدارة المشروع لتقديم منتجاتما وخدماها بنتائج وفوائد متفوقة يمكن أن تدوم لعملائها ومستخدميها، وإذا تمكنت المنظمة من تنفيذ إدارة المعرفة بفعالية، فهذا يُعتبر مفتاح النجاح في إدارة المشروع وبالتالي يمكن أن يحول المنظمة إلى التميز ويمنحها قيمة تنافسية مرموقة. وبالتالي، لكي تتمكن المنظمة من تحقيق المزيد من التغيير والنجاح؛ يجب دمج إدارة المعرفة مع إدارة المشروع للاستجابة السريعة لجمع المعلومات لحل مشاكل محددة وتبادل أصول المعرفة بفعالية وكفاءة، والعديد من المنظمات وقطاعات الأعمال تسعى إلى تطبيق إدارة المعرفة بمختلف عملياتا في مشاريعها، حيث تسعى هذه المنظمات من هذا التطبيق بالتأكيد إلى تققيق الربح وتخفيض تكلفة الانفاق بالإضافة إلى تقديم الجودة المطلوبة لضمان رضا العملاء عن الخدمات

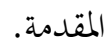

إنَّ إدارة المعرفة يجب أن تصبح جزءًا لا يتجزأ من العمل اليومي لكل مشروع، حيث أنه من الضروري دمج قواعد المعرفة في المشاريع حتى يتمكن الأشخاص المشاركون في المشروع من الجمع بين المساهمات الفردية فالمتعلقة بأهداف المشروع والمواءمة مع الأهداف الاستراتيجية للمنظمة.

واستنادًا إلى مراجعة الأدبيات ذات الصلة بأهمية العلاقة بين إدارة المعرفة وإدارة المشاريع فيّالاحظ أنه قد تم تطوير العديد من النماذج والأطر المقترحة لتوضيح الطريقة التي يمكن بها دمج إدارة المعرفة (KM) مع إدارة المشاريع (PM) للمساهمة في تعزيز نجاح المشروع. وهذه النماذج تتضمن بشكل أساسي تعزيز تطوير فريق المشروع، ومعرفة العملاء، والعلاقات من خلال ممارسات إدارة المعرفة المناسبة وتطبيقها في دورة حياة المشروع. كما أنَّ هذه النماذج تؤكد كذلك على أهمية الحصول على التغذية الراجعة من المشروعات لخلق المعرفة الابتكارية.

وبصورة مختصرة يُككن تلخيص أبرز نتائج الدراسة البحثية في النقاط التالية: 


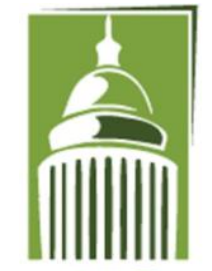

ARF
Global Proceedings Repository American Research Foundation

ISSN 2476-017X

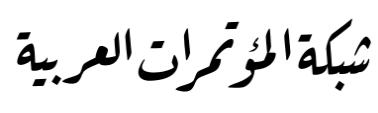

http://arab.kmshare.net/

Available online at http://proceedings.sriweb.org

1. إنَّ تطبيق المعرفة المناسبة والتي تتضمن الأدوات والتقنيات والعمليات والمهارات تتوثر بشكل كبير على نجاح المشروع، حيث أنَّ الهدف الأساسي من تنفي المشاريع هو ضمان اكتمال المشروع ضمن احتياجات المنظمة وضمن التكلفة المحددة والوقت المناسب والجودة المحددة للحصول على نتيجة فائية فعالة.

2. إدارة المعرفة تواجدت في المنظمات ومختلف قطاعات الأعمال لأنَّ لها تأثير كبير في دعم ثقافة المنظمة وتعزيز ميزتها التنافسية، وبالتالي من المختمل أن يكون لها علاقة وثيقة ومباشرة بإدارة المشاريع. 3. إدارة المعرفة تُمثل الممارسات المنطقية التي يُمكن من خلالها تحسين طريقة إدارة المشروع، وهذا يعني أنه سواء كانت المعرفة ضمنية أو صريحة فإنه يُمكن الحصول عليها ومشاركتها ونقلها بين أفراد المنظمة، وبالتالي ستستفيد منها كافة المنظمات وستعمل على المحافظة عليها واعادة استخدامها واسترجاعها عند الحاجة إليها مما سيُسهم بالضرورة في تحسين إدارة

$$
\text { المشروع. }
$$

4. إنَّ المنظمات التي تعتمد في إداراما للمنظمات البشرية على إدارة المعرفة من المحتمل أن تُحقق درجة عالية من التميز، حيث أها تعتمد في الضرورة على أدوات وطرق وأساليب يُمكن من خلالها نشر المعرفة وتوزيعها وتوليدها في الشركة، وهذا يؤكد

$$
\text { على الدور الفاعل لإدارة المعرفة ودمجها مع إدارة المشاريع. }
$$

5. يتضح التكامل بين إدارة المعرفة وإدارة المشاريع في تعزيز نجاح المشروع، وهذا يتضح من العملية التي يتم من خلالها انشاء

$$
\text { المعرفة ونقلها بين أعضاء الفريق بأحد أشكال المعرفة سواء المعرفة الضمنية أو الصريحة. }
$$

6. إنَّ العمل على تقييم المشروعات والحصول على التغذية الراجعة بشكل مستمر وتعزيز التواصل بين أفراد الفريق يُعتبر أمر

$$
\text { ضرورة لتعزيز فرصة نجاح المشروع. }
$$

7. يوجد العديد من العناصر المشتركة بين إدارة المعرفة وإدارة المشروع والتي لها تأثير كبير على نجاح المشروع، مثل الثقافة

$$
\text { التنظيمية والعمليات والتكنولوجيا وغيرها. }
$$




\section{Global Proceedings Repository \\ American Research Foundation}

ISSN 2476-017X

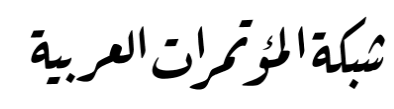

http://arab.kmshare.net/

AR F

Available online at http://proceedings.sriweb.org

بالتالي هذه النتائج تؤكد على وجود علاقة قوية بين إدارة المعرفة وإدارة المشاريع وتكاملهما في العديد من الجوانب، الأمر الذي يُعزّزِ من ضرورة تنفيذ المشاريع على اختلاف أنواعها في ظل إدارة العرفة بمختلف عملياتما. المقترحات

يوصي الباحث بالمقترحات التالية:

1. زيادة توعية العاملين في المشاريع بأهمية إدارة المعرفة وتبني عملياتا خلال دورة حياة المشروع من خلال عقد الورشات

التدريبية الخاصة بذلك.

2. تثقيف العاملين والمدراء بالوسائل والأساليب والممارسات الفعالة لإدارة المعرفة والتي يُمكن توظيفها في إدارة المشاريع.

3. ضرورة قيام المنظمة قبل تنفيذ المشروع بتحديد صلاحيات ومسؤوليات العاملين فيها وتحديد العلاقات بينهم بصورة

تضمن سير المشروع كما هو مخطط له.

4. تقييم المشاريع بعد الانتهاء من تنفيذها لقياس مستوى جودما ومدى نجاحها في تحقيق المطلوب وتحديد نقاط الضعف

التي ساهت في ضعف الأداء والعمل على تحسينها. 


\section{Global Proceedings Repository \\ American Research Foundation}

ISSN 2476-017X

Available online at http://proceedings.sriweb.org

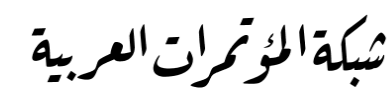

http://arab.kmshare.net/

أبو غانم، عبد الحميد (2002). إدارة المشروعات الصغيرة. طبعة أولى، دار الفجر للنشر والتوزيع، القاهرة.

بدر، يسرى (2010). تطوير مهارات مديري المدارس الثانوية في محافظات قطاع غزة في ضوء مفهوم إدارة المعرفة. رسالة

ماجستير، الجامعة الاسلامية، غزة.

تقرير هيئة الأمم المتحدة (2004). منهجية إدارة المعرفة: مقاربة تحريبية في قطاعات مركزية في دول الاسكوا الأعضاء.

نيويورك.

التلباني، غهاية؛ وبدير، رامز؛ زالرقب، يُمَّمَ (2015). متطلبات تطبيق إدارة المعرفة في الجامعات الفلسطينية بقطاع غزة. الجلة

$$
\begin{aligned}
& \text { الأردنية في إدارة الأعمال، المجلد 11، العدد2، ص443-480. } \\
& \text { حجازي، هيثم (2005). إدارة المعرفة،مدخل نظري. عمان: الأهلية للنشر والتوزيع. } \\
& \text { • ممود، خضير (2010). منظمة المعرفة. دار الصفاء للنشر والتوزيع، الطبعة الأولى، عمان. } \\
& \text { خير الدين، موسى (2012). إدارة المشاريع المعاصرة. دار وائل للنشر، الجامعة الأردنية، عمان، الأردن. }
\end{aligned}
$$

دروزة، سوزان (2008). العلاقة بين متطلبات إدارة المعرفة وعملياتا وأثرها على تميز الأداء المؤسسي دراسة تطبيقية في وزارة التعليم العالي الأردنية. رسالة ماجستير في إدارة الأعمال، جامعة الشرق الأوسك للدراسات العليا، كلية العلوم المالية والإدارية

دليل إدارة نطاق المشروعات، معهد التحديد القطاعي والمؤسساتي الأوروبي (ISMF). الاتحاد الأوروبي.

دهليز، خالد و عنبر، هشام (2017). أثر المجالات المعرفية لإدارة المشاريع على جودة المشاريع في المؤسسات الأهلية في

فلسطين. IUG Journal of Economics and Business (Islamic University of 


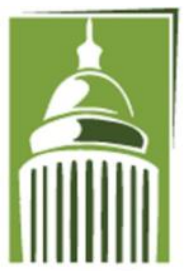

ARF
Global Proceedings Repository

American Research Foundation

ISSN 2476-017X

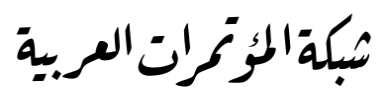

http://arab.kmshare.net/

Available online at http://proceedings.sriweb.org

دودين، أحمد (2012). إدارة المشاريع الهندسية، دار اليازوري للنشر، جامعة الزرقاء، عمان، الأردن.

الديري، علاء (2011). إدارة وتخطيط المشاريع الانشائية تأثير سوء التخطيط في مدة تنفيذ المشاريع الانشائية في إمارة دبي.

رسالة ماجستير، الجامعة الاسلامية، غزة، فلسطين.

رزوقي، نعيمة (2008). طريقة مستقبلية لذوي اختصاصي المعلومات في إدارة المعرفة. مجلة مكتبة الملك بفهد الوطنية،

مجلد9، عدد2. - na

صويص، راتب؛ وفلاق، تُحَّة؛ وبوقجاني، جناة وأبو حماد، أيمن (2011). عمليات إدارة المعرفة وتأُثيرها في تحقيق الميزة التنافسية دراسة حالة لمجموعة الاتصالات الأردنية (Orange). الجلة الأردنية في إدارة الأعمال، البجلد7، العدد4، ص

العامري، صالح (2007). الخطر في المشاريع وأساليب واستراتيجيات الاستجابة. مجلة جامعة البتراء، المجلد التاسع عشر، العدد الثاني، الأردن.

عبد العزيز، عماد (2008). إدارة مشتريات المشروع. أكاديمية الجمعيات الأهلية السعودية. عدس، عبد الرحن (1999). أساسيات البحث التربوي، الطبعة الأولى، دار الفرقان: عمان.

العلواني، حسن (2001). إدارة المعرفة المفهوم والمداخل النظرية. ورقة مقدمة إلى المؤتمر العربي الثاني في الإدارة "القيادة الابداعية في مواجهة التحديات المعاصرة للإدارة العربية" المنعقدة في المنظمة العربية للتنمية الإدارية التابعة لجامعة الدول العببية،

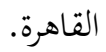

العلي، عابد (2011). دور التخطيط والرقابة في إدارة المشاريع باستخدام التحليل الشبكي. رسالة ماجستير، جامعة أبو بكر بلقايد، الجزائر. 


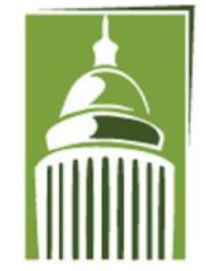

ARF
Global Proceedings Repository

American Research Foundation

ISSN 2476-017X

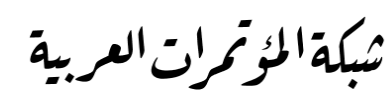

http://arab.kmshare.net/

Available online at http://proceedings.sriweb.org

عنبر، هشام (2016). دور المعايير الدولية لإدارة المشاريع في زيادة جودة المشروع في المسؤسسات الأهلية العاملة في قطاع غزة. رسالة ماجستير في إدارة الأعمال، كلية التجارة، الجامعة الاسلامية، غزة. الكبيسي، صلاح الدين (2005). إدارة المعرفة. المنظمة العربية للتنمية الإدارية. القاهرة، بحوث ودراسات.

مُحَّْ، لمياء (2012). تصميم خوارزمية جينية لايجاد المسار الحرج الأمثل لشبكة أعمال المشاريع. مجلة الرافدين لعلوم الحاسوب والرياضيات، المجلد التاسع، العدد الأول، العراق.

المقداد، طارق (2011). إدارة المشاريع الصغيرة الأساسيات والمواضيع المعاصرة. ورقة عمل، الاكاديمية العربية البريطانية. الميناوي، أيمن (2014). الوجيز العلمي لإدارة المشاريع. دار الأرقم للنشر والتوزيع، غزة، فلسطين. نايف، أسعد (2007). العلاقة بين إدارة المعرفة والمقدرة الجوهرية وأُرها على الأداء الاستراتيجي: دراسة استطلاعية تحليلية مقارنة لعينة من شركات وزارة الصناعة في مدينة بغداد. أطروحة دكتوراة، كلية الإدارة والاقتصاد، الجامعة المستنصرية، العراق. المراجع الأجنبية

- Al-Shafai, A. (2017). The relation between human resource management and organizational performance. Multi-Knowledge Electronic Comprehensive Journal For Education And Science Publications ( MECSJ ) ISSUE (3), p.256266.

- Akhavan, P., Adalati, M., Yazdi, S. and Hosnavi, R. (2010). The challenges of knowledge management portals application and implementation: An Iranian organizations case study. International Journal of Industrial Engineering Computations, 1(1):81-93. 


\section{Global Proceedings Repository \\ American Research Foundation}

ISSN 2476-017X

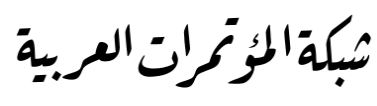

http://arab.kmshare.net/

Available online at http://proceedings.sriweb.org

- Bourdreau \& Couillard., (1999)“Systems integration and Knowledge Management”, Information System Management.

- Cope, R.F., Cope, R.F. \& Hotard, D.G. (2006),. Enhancing project management with knowledge Management principles, Allied Academies International Conference 2006, New Orleans, LA.

- Darling, M.S., (1996), Building the Knowledge Organization, Business Quartly, Vol.61, Issue.2.

- Duek, G., (2001), "Views of Knowledge are Human Views", IBM Systems Journal, Vol.40, No.4.

- Duffy, J., (2000), "Knowledge Management: To Be or Not to Be?", Information.

- Gudi, A. \& Becerra-Fernandez, I. (2006), Role of knowledge management in project management of complex systems organizations, NASA Knowledge Management and Successful Mission Operations Conference 2006, Houston, TX.

- Ismail, W.K.W., Nor, K.M. \& Marjani, T. (2009), The role of knowledge sharing practice in enhancing project success, Institute of Interdisciplinary Business Research, 1. 


\section{Global Proceedings Repository \\ American Research Foundation}

ISSN 2476-017X

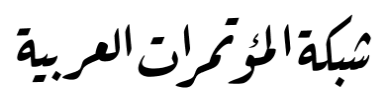

http://arab.kmshare.net/

Available online at http://proceedings.sriweb.org

- Khalid, F. (2017). The Impact of Poor Planning and Management on the Duration of Construction Projects: A Review. Multi-Knowledge Electronic Comprehensive Journal for Education and Science Publications (MECSJ) ISSUE (2), p. 161-182.

- Kerzner, H. (2009). Project Management - A Systems Approach to Planning, Scheduling, and Controlling, Wiley, New York.

- Lierni, P.C. \& Ribiere, V.M. (2008). The relationship between improving the management of projects and the use of KM, The Journal of Information and Knowledge Management Systems, vol 38, no. 1, 133-146.

- Mas-Machuca, M. (2014). The Role of Leadership: The Challenge of Knowledge Management and Learning in Knowledge-Intensive Organizations. Journal of Educational Leadership and Management, 2(1):97116.

- Mavodza, J. and Ngulube, P. (2012). Knowledge management practices at an institution of higher learning, SA Journal of Information Management. 14(1),8 pages.

- Modallal, A. (2012). The Implementation of Knowledge Management in the Palestinian Governmental Organizations and its Impact on the Level of 


\section{Global Proceedings Repository \\ American Research Foundation}

ISSN 2476-017X

Available online at http://proceedings.sriweb.org

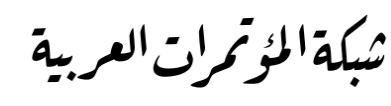

http://arab.kmshare.net/

Performance: Applied Study on the Council of Ministers, Unpublished Master Dissertation, Islamic University, Gaza- Palestine.

- Nonaka, I. Konno, N. (1998), "The Concept of "Ba" Building A foundation For Knowledge Creation", California Management Review, spring.

- Owen, J. (2008), Integrating knowledge management with programme management, Jennex, M.E. (ed.), Current Issues in Knowledge Management, IGI Global, New York, 132-148.

- Survary, M. (2005) Knowledge Management and Competition in Consulting Industry. Management Review, Vol. 41, No 2.

- The Project Management Institute (PMI) (2008). A Guide to the Project Management Body of Knowledge (PMBOK), Project Management Institute, Newtown Square, PA.

- Turner, J.R. (2009), the Handbook of Project-Based Management, Mc-Graw Hill, London.

- Yeong, A. and Lim, T. (2010). Integrating knowledge management with project management for project success. Journal of Project, Program \& Portfolio Management Vol 1 No 2 (2010) 8-19. 


\section{Global Proceedings Repository \\ American Research Foundation}

ISSN 2476-017X

Available online at http://proceedings.sriweb.org

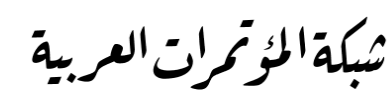

http://arab.kmshare.net/

- Levin, G. (2010), Knowledge mangement success equals project management success, PMI Global Congress 11 Oct 2010, Washington D.C.

- Polyaninova, T. (2011). Knowledge Management in a Project Environment:

Organisational CT and Project Influences. Dublin Institute of Technology. Vine, vol:41, iss:3.

- Al-Nady, B. A.-H. A., Al-Hawary, S. I. S., \& Alolayyan, M. N. (2016). The role of time, communication, and cost management on project management success: an empirical study on sample of construction projects customers in Makkah City, Kingdom of Saudi Arabia. International Journal of Services and Operations Management, 23(1), 76-112.

- Abdul Rasid, S. Z., Wan Ismail, W. K., Mohammad, N. H., \& Long, C. S. (2014). Assessing adoption of project management knowledge areas and maturity level: Case study of a public agency in Malaysia. Journal of Management in Engineering, 30(2), 264-271.

- Chauhan, D., \& Srivastava, P. (2014). Important Project Management knowledge areas for successful delivery of projects in Pharmaceutical Industry. International Journal of Current Research in Chemistry and Pharmaceutical Sciences, 1(5), 56-60. 


\section{Global Proceedings Repository \\ American Research Foundation}

ISSN 2476-017X

Available online at http://proceedings.sriweb.org

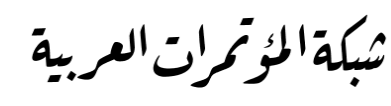

http://arab.kmshare.net/

AR F

- Heldman, K. (2009). PMP: Project Management Professional Exam Study

Guide (5 ed.). Indianapolis, Indiana: Wiley Publishing, Inc.

- Richman, L. (2011). Successful Project Management (3 ed.). New York, NY:

AMACOM, a division of the American Management Association

International.

- Wysocki, R. K. (2014). Effective Project Management: Traditional, Agile,

Extreme (7 ed.). USA: John Wiley \& Sons.

- Al-Zayyat, A., Khald, F., Tadros, I. and Al-Edwan, G. (2010). The Effect of Knowledge Management Processes on Project Management. IBIMA

Publishing, Journal of IBIMA Business Review, Vol. 2010 (2010), Article ID 826105, 6 pages.

- Oluikpe, P., Sohail, M. and Odhiambo, F. (2009). Towards a framework for Knowledge Management in project management. Loughborough University, Institutional Repository. International Journal of Knowledge Management Studies, 4(1), pp.18-46.

- Ritchie, P. \& Jorgensen, K. (2007). Project management knowledge management: moving from standards to leadership. Paper presented at PMI® 


\section{Global Proceedings Repository}

American Research Foundation

ISSN 2476-017X

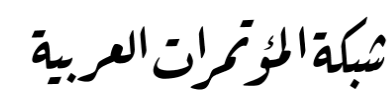

http://arab.kmshare.net/

Available online at http://proceedings.sriweb.org

Global Congress 2007-EMEA, Budapest, Hungary. Newtown Square, PA:

Project Management Institute.

- Levin, G. (2010). Knowledge management success equals project management success. Paper presented at PMI ${ }^{\circledR}$ Global Congress 2010 - North America, Washington, DC. Newtown Square, PA: Project Management Institute. 